\title{
Lewy body-like alpha-synuclein inclusions trigger reactive microgliosis prior to nigral degeneration
}

Megan F. Duffy ${ }^{1,2} \mathbb{D}$, Timothy J. Collier ${ }^{1,4}$, Joseph R. Patterson ${ }^{1}$ (D), Christopher J. Kemp ${ }^{1}$, Kelvin C. Luk ${ }^{5}$, Malú G. Tansey ${ }^{6}$, Katrina L. Paumier ${ }^{1,4}$, Nicholas M. Kanaan ${ }^{1,4}$ (D) D. Luke Fischer ${ }^{1,2,3}$ (D) Nicole K. Polinski ${ }^{1,2}$, Olivia L. Barth', Jacob W. Howe', Nishant N. Vaikath", Nour K. Majbour ${ }^{7}$, Omar M. A. El-Agnaf ${ }^{8}$ and Caryl E. Sortwell ${ }^{1,4^{*}}$ (D)

\begin{abstract}
Background: Converging evidence suggests a role for microglia-mediated neuroinflammation in Parkinson's disease (PD). Animal models of PD can serve as a platform to investigate the role of neuroinflammation in degeneration in PD. However, due to features of the previously available PD models, interpretations of the role of neuroinflammation as a contributor to or a consequence of neurodegeneration have remained elusive. In the present study, we investigated the temporal relationship of neuroinflammation in a model of synucleinopathy following intrastriatal injection of pre-formed alpha-synuclein fibrils (a-syn PFFS).

Methods: Male Fischer 344 rats $(N=114)$ received unilateral intrastriatal injections of a-syn PFFs, PBS, or rat serum albumin with cohorts euthanized at monthly intervals up to 6 months. Quantification of dopamine neurons, total neurons, phosphorylated a-syn (pS129) aggregates, major histocompatibility complex-II (MHC-II) antigen-presenting microglia, and ionized calcium-binding adaptor molecule-1 (Iba-1) immunoreactive microglial soma size was performed in the substantia nigra. In addition, the cortex and striatum were also examined for the presence of pS129 aggregates and MHC-II antigen-presenting microglia to compare the temporal patterns of pSyn accumulation and reactive microgliosis.

Results: Intrastriatal injection of a-syn PFFs to rats resulted in widespread accumulation of phosphorylated a-syn inclusions in several areas that innervate the striatum followed by significant loss ( $235 \%)$ of substantia nigra pars compacta dopamine neurons within 5-6 months. The peak magnitudes of a-syn inclusion formation, MHC-II expression, and reactive microglial morphology were all observed in the SN 2 months following injection and 3 months prior to nigral dopamine neuron loss. Surprisingly, MHC-II immunoreactivity in a-syn PFF injected rats was relatively limited during the later interval of degeneration. Moreover, we observed a significant correlation between substantia nigra pSyn inclusion load and number of microglia expressing MHC-II. In addition, we observed a similar relationship between a-syn inclusion load and number of microglia expressing MHC-II in cortical regions, but not in the striatum.

(Continued on next page)
\end{abstract}

\footnotetext{
* Correspondence: caryl.sortwell@hc.msu.edu

${ }^{1}$ Department of Translational Science and Molecular Medicine, Michigan

State University, 400 Monroe Avenue NW, Grand Rapids, MI 49503-2532, USA

${ }^{4}$ Mercy Health Hauenstein Neuroscience Medical Center, Grand Rapids, MI,

USA

Full list of author information is available at the end of the article
}

(c) The Author(s). 2018, corrected publication May/2018. Open Access This article is distributed under the terms of the Creative Commons Attribution 4.0 International License (http://creativecommons.org/licenses/by/4.0/), which permits unrestricted use, distribution, and reproduction in any medium, provided you give appropriate credit to the original author(s) and the source, provide a link to the Creative Commons license, and indicate if changes were made. The Creative Commons Public Domain Dedication waiver (http://creativecommons.org/publicdomain/zero/1.0/) applies to the data made available in this article, unless otherwise stated. 


\begin{abstract}
(Continued from previous page)
Conclusions: Our results demonstrate that increases in microglia displaying a reactive morphology and MHC-II expression occur in the substantia nigra in close association with peak numbers of pSyn inclusions, months prior to nigral dopamine neuron degeneration, and suggest that reactive microglia may contribute to vulnerability of SNc neurons to degeneration. The rat a-syn PFF model provides an opportunity to examine the innate immune response to accumulation of pathological a-syn in the context of normal levels of endogenous a-syn and provides insight into the earliest neuroinflammatory events in PD.
\end{abstract}

Keywords: Neuroinflammation, Parkinson's disease, Animal models, Synucleinopathy, Microglia, Major-histocompatibility complex-II, Neurodegeneration, Selective vulnerability

\section{Background}

The etiology of Parkinson's disease (PD) is stochastic: a culmination of aging-related changes in brain environment, genetic predispositions, and environmental insults that result in accumulation of alpha-synuclein ( $\alpha$-syn) inclusions (i.e., Lewy bodies) and degeneration of nigrostriatal dopamine neurons $[1,2]$. Converging evidence suggests a role for microglia-mediated neuroinflammation in human PD. This theory is supported by observations of increased inflammatory cytokines in both $\mathrm{PD}$ patient cerebral spinal fluid (CSF) and plasma [3, 4] and in the patient brain as longitudinal PET imaging has demonstrated early and sustained microglial activation in the basal ganglia [5]. Furthermore, postmortem analyses in PD patients revealed increased expression of inflammatory markers such as human leukocyte antigen (HLA-DR), major histocompatibility complex-II (MHCII), phagocytic marker CD68, intercellular adhesion molecule-I (ICAM-1), and integrin adhesion molecule (LFA-1) in the substantia nigra [6, 7]. However, a drawback of biofluid and postmortem PD brain samples is that they only provide a static snapshot of events within a longitudinal cascade of PD pathophysiology. This is especially problematic as the overwhelming majority of $\mathrm{PD}$ patient samples are collected from individuals who have likely harbored PD-related pathology for decades before, if also not after, diagnosis [8]. This confounds interpretations of the role of neuroinflammation in degeneration in PD and prevents the understanding as to whether neuroinflammation participates as a contributor to nigral degeneration or is simply an artifact of cell death.

One of the most consistent observations in postmortem PD tissue is an increase in the number of microglia expressing MHC-II (HLA-DR in humans [7, 9, 10]), a cell surface protein on antigen-presenting cells which is necessary for $\mathrm{CD} 4+\mathrm{T}$ cell infiltration. More recently, gene expression changes related to inflammation, including an upregulation of MHC-II, have also been noted in incidental Lewy body disease subjects (Braak stages 1-3 $[10,11])$. Additionally, a variant in the HLA-DR gene which encodes for MHC-II is associated with amplified risk for development of PD after pesticide exposure [12]. Increased MHC-II is often concurrently upregulated with genes for proinflammatory cytokines such as tumor necrosis factor (TNF) and interleukin-1 beta (IL-1 $\beta$ ) [13]. Moreover, decreased MHC-II expression was shown to attenuate downstream secretion of proinflammatory cytokines [14, 15]. Taken together, it is likely that MHC-II is most closely associated with a proinflammatory phenotype in microglia and may play a contributory role in nigral degeneration in PD. However, while the concept that MHC-II expression on microglia is increased in PD patients is not novel [9], the temporal pattern of observed increases in MHC-II in relation to $\alpha$-syn aggregation and/or nigrostriatal degeneration has been unable to be systematically examined.

Animal models of PD can serve as platforms to investigate the role of neuroinflammation in PD-related cell death and dysfunction. The neuroinflammatory consequences of nigral degeneration and/or $\alpha$-syn aggregation have been examined previously in various models, including but not limited to, neurotoxicant models (6-hydroxydopamine (6-OHDA) [16, 17]; 1-methyl-4phenyl-1,2,3,6-tetrahydropyridine (MPTP) [18, 19]); transgenic models expressing human wild-type or mutant $\alpha$-syn (A503T, A30P [20-22]) and viral vector-mediated overexpression of human wild-type or mutated $\alpha$-syn in the nigrostriatal system [22-28]. However, certain characteristics of these models limit interpretations regarding the specific initiator of the neuroinflammation observedsynuclein inclusions and/or degeneration. Neurotoxicant models (6-OHDA, MPTP) rarely exhibit $\alpha$-syn pathology $[18,29]$. Transgenic models generally do not recapitulate marked nigrostriatal degeneration despite widespread, $\alpha$-syn pathology [21, 30]. Whereas a robust inflammatory response is observed in association with the elevated $\alpha$-syn levels, aggregates, and nigral degeneration in viral vector-based $\alpha$-syn overexpression models [22-28, 31-33], the contribution of supraphysiological $\alpha-$ syn levels or the $\alpha$-syn species difference (human $\alpha$-syn expressed in rat or mouse) to the neuroinflammatory response is unclear. Importantly, in human sporadic PD, total $\alpha$-syn levels are not increased; rather, phosphorylation 
and the ratio of soluble to insoluble $\alpha$-syn increases over time [34-36].

An alternative model of the key features of human sporadic PD such as (1) protracted development of $\alpha$-syn inclusions under conditions of (2) normal expression levels of endogenous $\alpha$-syn during an interval that (3) precedes significant nigrostriatal degeneration would offer distinct advantages and allow the time course and potential impact of neuroinflammation to be delineated. Recently, our lab has characterized a rat model of PD that recapitulates this sequence of events, extending previous findings in mice $[37,38]$. In this model, nigrostriatal synucleinopathy is induced by intrastriatal injection of sonicated pre-formed $\alpha$-syn fibrils ( $\alpha$-syn PFFs) into wild-type rats [37, 39]. The fibrils act as seeds to template and trigger normal levels of endogenous $\alpha$-syn to accumulate into misfolded hyperphosphorylated, pathological $\alpha$-syn (Fig. 1a). The initial injection of $\alpha$-syn PFFs per se does not directly cause toxicity, given that $\alpha$-syn pathology and nigral degeneration do not occur in $\alpha$-syn knockout animals injected with PFFs [38]. In this model, we observe a widespread accumulation of intraneuronal Lewy neuritelike and Lewy body-like inclusions of phosphorylated $\alpha$-syn (pSyn) in areas that innervate the striatum. Importantly, the accumulation of intracellular pSyn is gradual and results in loss of striatal dopamine and metabolites in addition to $\sim 40 \%$ loss of SNc dopamine neurons over 6 months [37]. Thus, the synucleinopathy produced in the $\alpha$-syn PFF model provides a unique opportunity to examine the neuroinflammatory consequences of $\alpha$-syn inclusion accumulation in the context of normal levels of endogenous, intracellular $\alpha$-syn. In the present study, we systematically investigated the temporal profile of Lewy body-like phosphorylated $\alpha$-syn inclusion load, reactive microglial morphology, MHC-II antigen presentation, and degeneration in the SN. Importantly, we observe reactive microglia and increased microglial MHC-II expression in association with peak load of SNc pSyn inclusions months prior to degeneration, suggesting that neuroinflammation may contribute to nigrostriatal degeneration.

\section{Methods}

\section{Animals}

Young adult ( 2 months), male Fischer344 rats $(n=114)$ were used in this study. All animals were provided food and water ad-libitum and housed at the AAALACapproved Van Andel Research Institute vivarium. All procedures were approved and conducted in accordance with Institute for Animal Use and Care Committee (IACUC) at Michigan State University.

\section{Preparation of a-syn PFFs and verification of fibril size}

Purification of recombinant, full-length mouse $\alpha$-syn and in vitro fibril assembly was performed as previously described [39-41]. Prior to sonication, $\alpha$-syn fibrils were assessed to verify lack of contamination (LAL Assay $(\sim 1$ endotoxin units/mg), high molecular weight (sedimentation assay), beta sheet conformation (thioflavin $\mathrm{T}$ ), and structure (electron microscopy). Prior to injection, PFFs were thawed, diluted in sterile Dulbecco's PBS (DPBS, $2 \mu \mathrm{g} / \mu \mathrm{l}$ ), and sonicated at room temperature using an ultrasonicating homogenizer (300VT; Biologics, Inc., Manassas, VA) with the pulser set at $20 \%$ and power output at $30 \%$ for 60 pulses at $1 \mathrm{~s}$ each. Following sonication, a sample of the PFFs was analyzed using transmission electron microscopy (TEM). Formvar/carbon-coated copper grids (EMSDIASUM, FCF300-Cu) were washed twice with $\mathrm{ddH}_{2} \mathrm{O}$ and floated for $1 \mathrm{~min}$ on a 10- $\mu$ l drop of sonicated $\alpha$-syn fibrils diluted 1:20 with DPBS. Grids were stained for $1 \mathrm{~min}$ on a drop of $2 \%$ uranyl acetate aqueous solution; excess uranyl acetate was wicked away with filter paper and allowed to dry before imaging. Grids were imaged on a JEOL JEM-1400 transmission electron microscope. The length of over 500 fibrils per sample was measured to determine average fibril size. The mean length of sonicated mouse $\alpha$-syn PFFs was estimated to be $51.22 \pm 1.31 \mathrm{~nm}$, well within the optimal fibril length previously reported to result in seeding of endogenous phosphorylated $\alpha$-syn inclusions in vitro and in vivo (Fig. 1b, c) [42].

\section{Intrastriatal injections}

Sonicated PFFs were kept at room temperature during the duration of the surgical procedures. All rats were deeply anesthetized with isoflurane received two $2-\mu \mathrm{l}$ unilateral intrastriatal injections $(4 \mu \mathrm{l}$ total; $\mathrm{AP}+1.6$, $\mathrm{ML}+2.4, \mathrm{DV}-4.2 ; \mathrm{AP}-1.4, \mathrm{ML}+2.0, \mathrm{DV}-7.0$ from the skull) either of sonicated mouse $\alpha$-syn PFFs $(2 \mu \mathrm{g} / \mu \mathrm{l}$ as described previously [37]) or an equal volume of DPBS at a rate at $0.5 \mu \mathrm{l} / \mathrm{min}$ ( $n=6$ per treatment per time point). Injections were administered made using a pulled glass needle attached to a 10- $\mu$ l Hamilton syringe. After each injection, the needle was left in place for $1 \mathrm{~min}$, retracted $0.5 \mathrm{~mm}$, left in place for an additional $2 \mathrm{~min}$, and then slowly withdrawn. Animals were monitored post-surgery and euthanized at predetermined time points $(14,30,60,90,120$, 150, and 180 days; Fig. 1). In a subsequent experiment, rats received two 2- $\mu \mathrm{l}$ unilateral intrastriatal injections either of mouse $\alpha$-syn PFFs $2 \mu \mathrm{g} / \mu \mathrm{l}$, DPBS, or rat serum albumin (RSA, Sigma-Aldrich, St. Louis, MO; 9048-46-8; $2 \mu \mathrm{g} / \mu \mathrm{l}$ ) at the identical coordinates and were euthanized at 2 months postinjection ( $n=6$ per treatment).

\section{Immunohistochemistry}

All animals were euthanized via pentobarbital overdose $(60 \mathrm{mg} / \mathrm{kg})$ and intracardially perfused with heparinized $0.9 \%$ saline followed by cold $4 \%$ paraformaldehyde in $0.1 \mathrm{M} \mathrm{PO}_{4}$. Brains were extracted and postfixed in $4 \%$ PFA for $48 \mathrm{~h}$ and placed in 30\% sucrose until they sunk. 


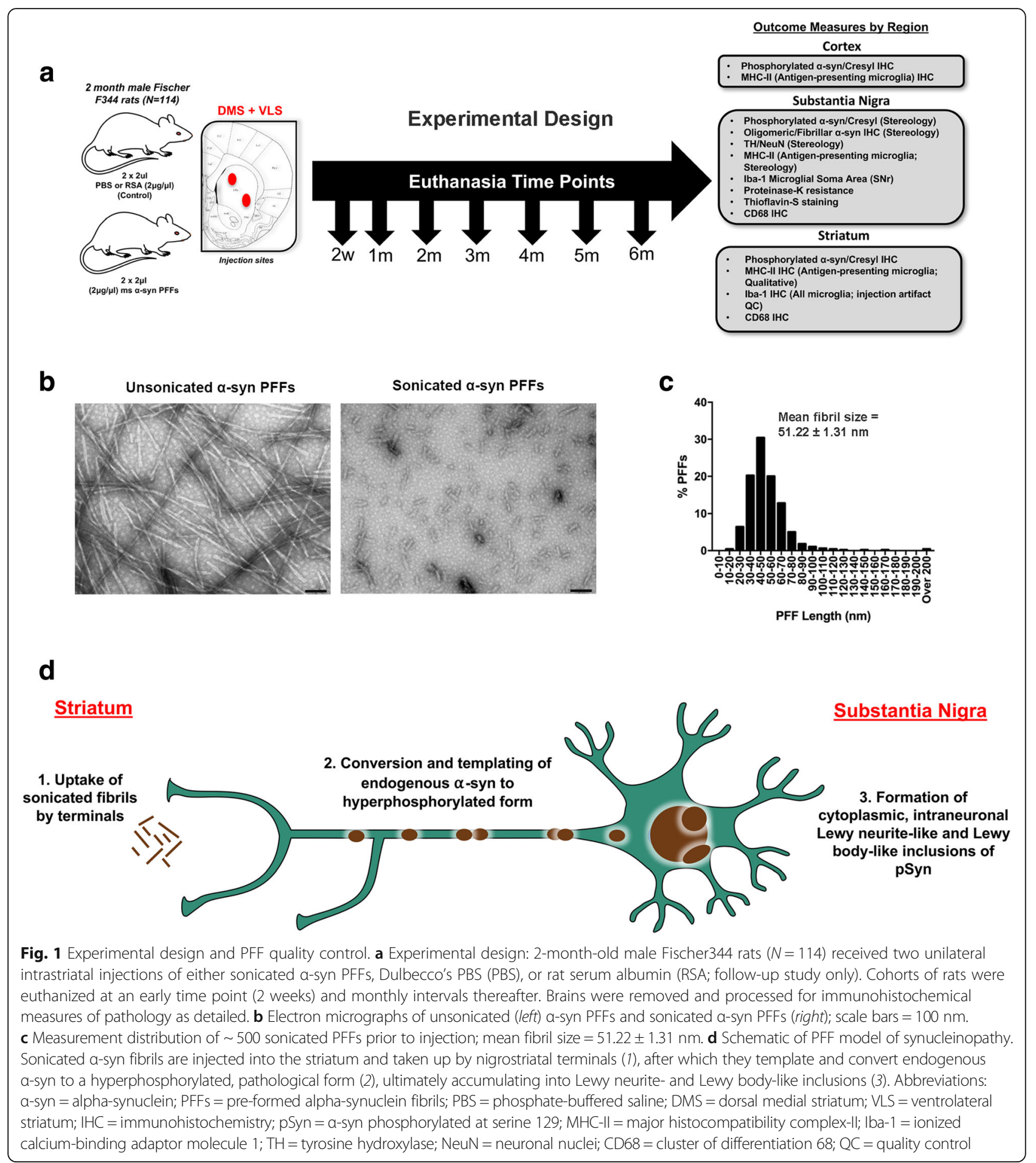

For sectioning, brains were frozen on a sliding microtome and cut at $40 \mu \mathrm{m}$. Free-floating sections (1:6 series) were transferred to $0.1 \mathrm{M}$ tris-buffered saline (TBS). Following the washes, endogenous peroxidases were quenched in 3\% $\mathrm{H}_{2} \mathrm{O}_{2}$ for $1 \mathrm{~h}$ and rinsed in TBS. Sections were blocked in $10 \%$ normal goat serum/0.5\% Triton X-100 in TBS (NGS, Gibco; Tx-100 Fischer Scientific) for $1 \mathrm{~h}$. Following the blocking, sections were immunolabeled with primary antibodies: mouse anti- $\alpha$-syn fibrils/oligomers $(\mathrm{O} 2 ; 1: 5000$ [43]) or mouse anti $\alpha$-syn fibrils (F2; 1:5000 [43]), pan rabbit-anti $\alpha$-syn (Abcam, Cambridge, MA; AB15530, 1:1000), mouse anti-phosphorylated $\alpha$-syn at serine 129 (pSyn, 81A; Abcam, Cambridge, MA; AB184674; 1:10,000), rabbit anti-tyrosine hydroxylase (TH; Millipore, 
Temecula, CA; MAB152, 1:4000), rabbit anti-ionized calcium-binding adaptor molecule-1 (Iba-1; Wako, Richmond, VA; 019-19741, 1:1000), mouse anti-neuronal nuclei (Neu-N; Millipore, Temecula, CA; MAB 377, 1:5000), or mouse anti-rat major histocompatibility complex-II for antigen-presenting microglia (MHC Class II RT1B clone OX-6, Bio-Rad, Hercules, CA; MCA46G, 1:5000) overnight in $1 \% \mathrm{NGS} / 0.5 \% \mathrm{Tx}-100 / \mathrm{TBS}$ at $4{ }^{\circ} \mathrm{C}$. Following the washes, sections were incubated in biotinylated secondary antibodies (1:500) against mouse (Millipore, Temecula, CA; AP124B) or rabbit IgG (Millipore, Temecula, CA; AP132B) followed by washes in TBS and 2-h incubation with Vector $A B C$ standard detection kit (Vector Laboratories, Burlingame, CA; PK-6100). Labeling for pSyn, MHC-II, and TH was visualized by development in 0 .

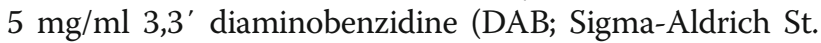
Louis, MO; D5637-10G) and $0.03 \% \mathrm{H}_{2} \mathrm{O}_{2}$. For dual brightfield visualization of Neu-N and Iba-1, sections were developed according to the manufacturer's instructions using the Vector ImmPACT DAB Peroxidase (Vector Labs, Burlingame, CA; SK-4605) and ImmPACT VIP Peroxidase (Vector Labs, Burlingame, CA; SK-4105) kits, respectively. Slides were dehydrated in ascending ethanol series and then xylenes before coverslipping with Cytoseal (Richard-Allan Scientific, Waltham, MA). A subset of pSyn-labeled sections were also counterstained with cresyl violet for quantification of intraneuronal pSyn inclusions in the SNc.

\section{RNAscope in situ hybridization for Iba-1 and MHC-II IHC}

Forty-micrometer-thick striatal tissue sections were incubated in pretreat 1 from the RNAscope Pretreatment Kit (Advanced Cell Diagnostics, Hayward, CA; 310020) for $1 \mathrm{~h}$. Sections were washed in TBS and then mounted on VistaVision HistoBond slides (VWR, Randor, PA; 16004-406) and placed on slide warmer at $60{ }^{\circ} \mathrm{C}$ overnight. Slides were then incubated for $10 \mathrm{~min}$ in pretreat 2 at $99{ }^{\circ} \mathrm{C}$ and washed twice in water. Tissue was outlined with Pap Pen (Abcam, Cambridge, UK; ab2601), incubated with pretreat 3 in a hybridization oven at $40{ }^{\circ} \mathrm{C}$ for $15 \mathrm{~min}$, washed twice in water, and incubated with the probe for AIF1 (Iba1; Advanced Cell Diagnostics, Hayward, CA; 457731) for $2 \mathrm{~h}$ in the hybridization oven at $40{ }^{\circ} \mathrm{C}$. Six amplification steps with the amplification buffers (Advanced Cell Diagnostics, Hayward, CA; 320600) were then performed in alternating 30- and 15-min incubation intervals in the hybridization oven per manufacturer instructions. Tissue was developed using the supplied DAB reagent (Advanced Cell Diagnostics, Hayward, CA; 320600). Tissue was then counterstained for MHC-II (RT1B clone OX-6, Bio-Rad, Hercules, CA; MCA46G, 1:500) in a hybridization chamber, following the same procedures as detailed for other immunohistochemical stains with the exception that the Vector SG reagent (Vector Laboratories, Burlingame, CA) was used as the chromogen. Slides were rinsed in TBS and coverslipped with Cytoseal 60. Images were taken on a Nikon Eclipse 90i microscope with a QICAM camera (QImaging, Surrey, British Colombia, Canada).

\section{Quantification of TH, NeuN, pSyn, and MHC-II immunoreactive profiles}

Microbrightfield (MBF) Stereoinvestigator (MBF Bioscience, Williston, VT) was used to estimate the total population of THir and NeuNir neurons to determine the time course of TH phenotype loss and overt nigral degeneration. Contours were drawn around the SNc using the $\times 4$ objective on every sixth section through the rostrocaudal axis $(9-10$ sections). A series of counting frames $(50 \mu \mathrm{m} \times 50 \mu \mathrm{m})$ was systematically and randomly distributed over grid $(183 \mu \mathrm{m} \times 112 \mu \mathrm{m})$ placed over the SNc, allowing for quantification of approximately $20 \%$ of the SNc. An investigator blinded to experimental conditions counted THir and NeuNir cells using the optical fractionator probe with a $\times 60$ oil immersion objective. Markers were placed on each THir or NeuNir cell in a 1-2- $\mu \mathrm{m}$ z-stack within the counting frame. Between 50 and 500 objects were counted to generate stereological estimates of the total cell population. The total population estimate was calculated using optical fractionator estimates, and variability within animals was assessed via the Gunderson coefficient of error $(<0.1)$. Due to heterogeneity in the distribution of both pSyn and MHC-II immunoreactive profiles within the SN, total enumeration rather than counting frames was used for quantification. Neurons with intraneuronal pSyn inclusions were defined as profiles of dark, densely stained pSyn immunoreactivity within cresyl violetpositive neurons. Contours were drawn around the $\mathrm{SNc}$ using the $\times 4$ objective on every sixth section through the entire rostrocaudal axis of the SNc (9-10 sections). pSyn inclusions and MHC-IIir microglia were then systematically counted within each contour using the $\times 20$ objective. Numbers represent the raw total number of pSyn inclusions or MHC-IIir microglia per animal multiplied by 6 to extrapolate the population estimate.

\section{Microglial soma area analysis}

Forty-micrometer-thick nigral tissue sections (1:6 series) from animals injected with $\alpha$-syn PFFs, RSA, or and DPBS 2 months and 6 months following injection were dual labeled for NeuN and Iba-1 as described above to distinguish the SNc from the SNr. The three nigral sections adjacent to the sections containing the most $\mathrm{pSyn}$ inclusions were identified. z-stack images of the ipsilateral and contralateral $\mathrm{SNr}$ bordering the $\mathrm{SNc}$ were taken on a Nikon Eclipse 90i microscope with a QICAM camera (QImaging, Surrey, British Colombia, Canada) using 
the $\times 20$ objective and analyzed with Nikon Elements AR (version 4.50.00, Melville, NY). Using the auto-detect feature, each Iba-1ir soma's border was outlined and adjusted accordingly to obtain an accurate quantification of area of the soma, excluding any processes. All microglia in the field of view of each z-stack per section, per rat were quantified with total number of microglia per rat calculated (100-250). Data are expressed as mean Iba-1ir soma area per treatment group. Soma measurements for all microglia per treatment were also grouped into $10 \mu \mathrm{m}$ bins and expressed as a percentage of total microglia counted.

\section{Thioflavin-S staining}

1:12 series was washed in TBS and subsequently mounted on subbed slides to dry $(\sim 1 \mathrm{~h})$. Slides were incubated in $0.5 \% \mathrm{KMnO}_{4}$ in TBS for $25 \mathrm{~min}$, followed by five washes in TBS. Sections were destained in $0.2 \%$ $\mathrm{K}_{2} \mathrm{~S}_{2} \mathrm{O}_{5} / 0.2 \%$ oxalic acid in TBS for 3 min followed by incubation in $0.0125 \%$ thioflavin-S in $40 \% \mathrm{EtOH} / \mathrm{TBS}$ for $3 \mathrm{~min}$ and differentiated in $50 \% \mathrm{EtOH}$ for $15 \mathrm{~min}$. Sections were rinsed first in TBS and then $\mathrm{ddH}_{2} \mathrm{O}$ before coverslipping with VECTASHIELD Mounting Medium for fluorescence.

\section{Proteinase-K digestion}

1:12 nigral series was washed in TBS. A subset of free floating tissue sections was treated with $10 \mu \mathrm{g} / \mathrm{ml}$ proteinase K (Invitrogen, Carlsbad, CA; 25530015) for $30 \mathrm{~min}$ at room temperature, followed by three washes in TBS and four washes in TBS-Tx. Sections were then processed for pan $\alpha$-syn immunohistochemistry (rabbit anti- $\alpha$-syn, Abcam, Cambridge, UK; AB15530) as described above, mounted on subbed slides, dehydrated to xylenes, and coverslipped.

\section{Statistics}

Statistical analyses were performed using IBM SPSS Statistics (IBM, Armonk, NY) or GraphPad Prism (La Jolla, CA). Statistical significance for all cases was set at $p<0.05$. Statistical outliers were assessed using the Absolute Deviation from the Median (ADAM) method using the "very conservative" criterion [44]. To compare numbers of $\mathrm{O} 2$ vs. F2 immunoreactive cells (Fig. 4), THir and NeuNir neurons (Fig. 5), pSyn $\alpha$-syn inclusions (Fig. 6), MHC-IIir microglia (Fig. 5), and Iba-1ir microglia number and size (Fig. 7), a one-way ANOVA with Tukey's post hoc analyses was used. Correlation analysis was conducted to investigate the relationship between ipsilateral and contralateral THir neurons (Fig. 5) and between MHC-IIir and pSyn $\alpha$-syn inclusions (Fig. 6).

\section{Results}

Sonicated a-syn PFFs are the optimal size for pathology induction in vivo

Prior to intrastriatal injection of mouse $\alpha$-syn PFFs, we investigated the size of the PFFs following sonication using transmission electron microscopy (TEM; Fig. 1b, c). The mean length of sonicated mouse $\alpha$-syn PFFs was estimated to be $51.22 \pm 1.31 \mathrm{~nm}$, well within the optimal fibril length previously reported to result in seeding of endogenous phosphorylated $\alpha$-syn inclusions (Fig. 1d, schematic) in vitro and in vivo [42].

\section{Unilateral intrastriatal injection of a-syn PFFs induces widespread Lewy-like pathology}

In our previous work [37], we reported that unilateral intrastriatal injection of mouse $\alpha$-syn PFFs results in phosphorylated $\alpha$-syn (pSyn) intraneuronal accumulations in several areas that innervate the striatum [45], most prominently the frontal (primary motor and somatosensory, layer 5) and insular cortices, amygdala, and SNc. Over time, accumulations increase in number in these regions. In the present study, we observed an identical pattern of pSyn accumulation in rats injected with mouse $\alpha$-syn PFFs. Specifically, we observe abundant pSyn pathology bilaterally in cortical regions (layers $2 / 3$ of the secondary motor area, insular cortex, and orbital areas; Fig. 2a). In contrast, we observed unilateral pSyn accumulation in the $\mathrm{SNc}$ ipsilateral to the injected striatum and complete absence of pSyn aggregates in animals injected with an equal volume of PBS or equal volume and concentration of RSA (Additional file 1: Figure S1).

Accumulation of pSyn inclusions followed a distinct temporal pattern depending on the region examined. At 2 months postinjection (p.i.), we observed abundant soma and neuritic pSyn inclusions bilaterally in the agranular insular cortex that persisted over the course of 6 months (Additional file 2: Figure S2A). Abundant pSyn accumulations were observed within the ipsilateral SNc at 2 months p.i., which remained ipsilateral and decreased in number over the course of 6 months (Additional file 2: Figure S2D-F). The abundance of pSyn inclusions in the striatum followed an opposite pattern (Additional file 2: Figure S2G-I). We observed relatively sparse pSyn inclusions in the striatum at 2 months p.i. that were primarily restricted to neurites. At 4 and 6 months p.i., the number of pSyn accumulations in striatal somata increased in abundance and also were observed in the contralateral striatal hemisphere (Additional file 2: Figure $\mathrm{S} 2 \mathrm{H}-\mathrm{I}$ ).

\section{a-Syn inclusions in the SNc exhibit oligomeric, fibrillary conformations, and Lewy body-like characteristics}

The oligomeric form of $\alpha$-syn is proposed to be one of the toxic species [43, 46-48]. We further characterized the nature of pSyn inclusions within the SNc at 1 month 

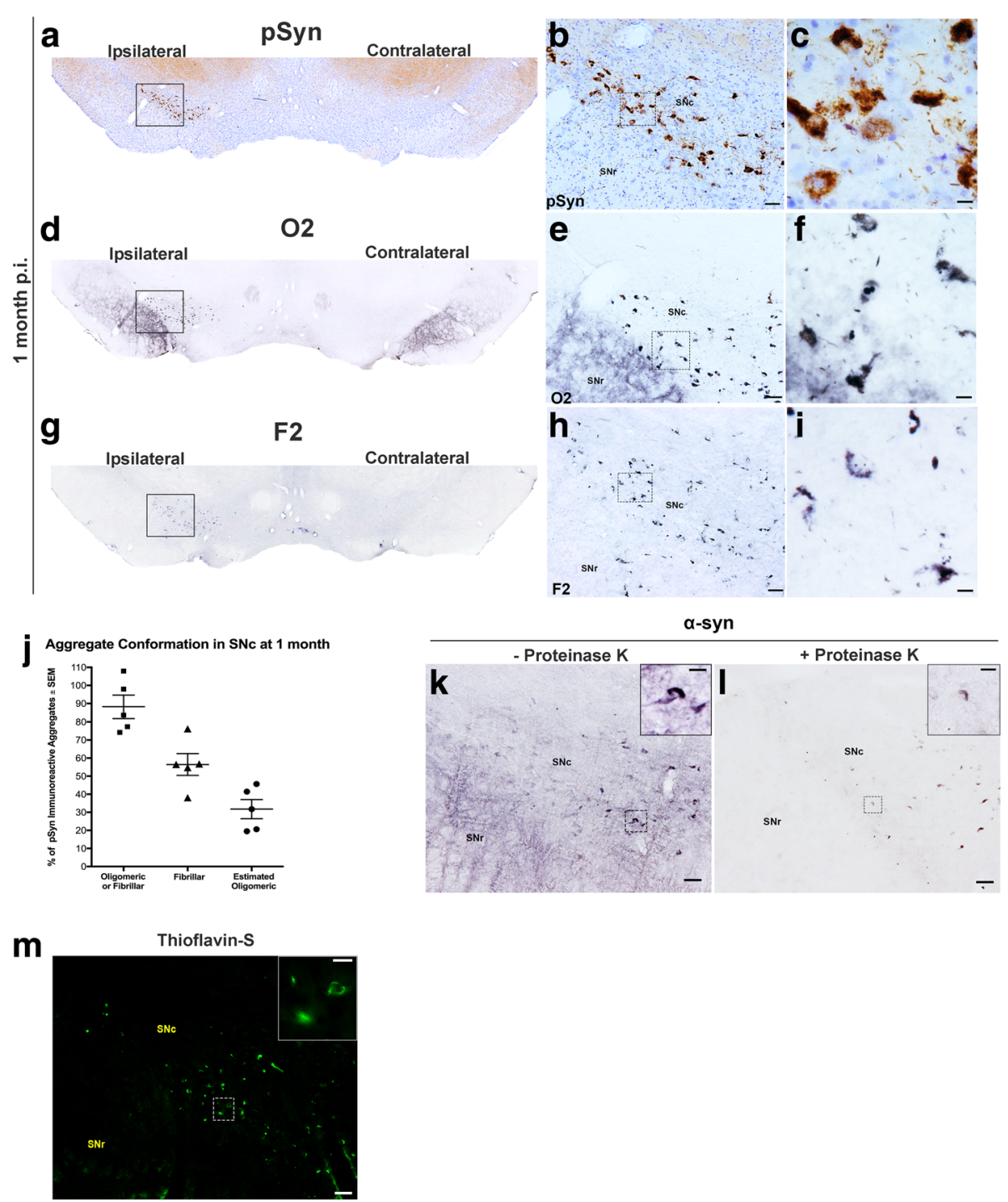

Fig. 2 a-Syn inclusions in the SNc exhibit oligomeric and fibrillary conformations and Lewy body-like characteristics. a-c Representative images of Lewy-body-like intraneuronal pSyn inclusions in the substantia nigra pars compacta (SNc) at 1 month p.i. show pathology is localized to the ipsilateral SNc. d-f Adjacent SN tissue sections stained for O2 (oligomeric/fibrillar a-syn conformation specific) and F2 (g-i) (fibrillar-specific conformation) a-syn reveal that many intraneuronal inclusions possess mature, fibrillar inclusions of a-syn. $\mathbf{j}$ Percent of pSyn inclusions with either oligomeric/fibrillar (O2) or predominantly fibrillar (F2) immunoreactivity and estimated proportion of pSyn inclusions that are oligomeric only. Data represent mean \pm SEM. Scale bars $(\mathbf{b}, \mathbf{e}, \mathbf{h})=50 \mu \mathrm{m},(\mathbf{c}, \mathbf{f}, \mathbf{i})=10 \mu \mathrm{m}$. $\mathbf{k}$ Endogenous a-syn immunoreactivity in the SNc and SNr. I Adjacent tissue sections exposed to proteinase-K reveal the absence of soluble a-syn in the $\mathrm{SNr}$ and the presence of insoluble, neuronal inclusions of a-syn in the SNc. $\mathbf{m}$ Thioflavin-S fluorescence of amyloid structure present in SNc neurons. Scale bars $(\mathbf{k}, \mathbf{l}, \mathbf{m})=50 \mu \mathrm{m}$, (insets) $=25 \mu \mathrm{m}$. Abbreviations: a-syn = alpha-synuclein; p.i. = postinjection; pSyn = a-syn phosphorylated at serine 129; O2=oligomeric/fibrillar a-syn antibody; F2= fibrillar a-syn only antibody; $\mathrm{SNc}=$ substantia nigra pars compacta; SNr = substantia nigra pars reticulata; $\mathrm{SEM}=$ standard error of the mean

p.i. using conformation-specific antibodies for oligomeric/fibrillar $\alpha$-syn (O2) or fibrillar-predominant $\alpha$-syn (F2) and compared that with immunoreactivity to pSyn (Fig. 2a-i [43]). When adjacent sections were quantified using unbiased stereology, we observed that $88.2 \pm 6.4 \%$ of pSyn immunoreactive inclusions exhibited either an oligomeric or fibrillary conformation (O2 only). Furthermore, $56.4 \pm 6.04 \%$ of nigral pSyn inclusions was detected as predominately mature, fibrillar aggregates (F2 only) with an estimated $31.7 \pm 5.9 \%$ of inclusions suggested to be in an oligomeric conformation (O2 only minus F2 only; Fig. 2j). $\alpha$-Syn inclusions in the SNc 2 months p.i. displayed Lewy body-like characteristics $[49,50]$, including resistance to proteinase- $\mathrm{K}$ digestion (Fig. 2i, k, l) as well as markers for $\beta$-sheet structure as detected by thioflavin-S (Fig. $2 \mathrm{~m}$ ). Collectively, these results suggest that intrastriatal injection of mouse $\alpha$-syn PFFs triggers pathological conversion of endogenous 
$\alpha$-syn to phosphorylated, oligomeric, and fibrillary conformations in the $\mathrm{SNc}$ that ultimately result in insoluble, amyloid inclusions resembling Lewy bodies.

\section{PFF-induced synucleinopathy induces significant bilateral loss of SNc neurons}

We previously observed that unilateral intrastriatal mouse $\alpha$-syn PFF injections to rats resulted in bilateral nigrostriatal degeneration of THir SNc neurons within 6 months [37]. To validate this finding in our present cohort, we conducted unbiased stereology of THir SNc neurons at 2, 4, 5, and 6 months p.i. in $\alpha$-syn PFF- and PBS-injected rats. Injection of PBS did not result in significant loss of THir SNc neurons at any time point $\left(F_{(7,18)}=1.991, p>0.05\right)$; thus, PBS-injected time points were combined for comparison to PFF-injected rats between identical hemispheres (ipsilateral $\mathrm{PBS}=12,518 \pm 554$; contralateral PBS $11,577 \pm 536$ ). Similar to our previous studies, we observed significant, bilateral reduction $(\sim 35 \%)$ in SNc THir neurons (Fig. 3a-e). Specifically, the number of SNc THir neurons ipsilateral to $\alpha$-syn PFF injection at both $5(8227 \pm 1015)$ and 6 months $(8851 \pm 1148)$ p.i. was significantly reduced compared to PBS control rats $\left(F_{(5,36)}=4.297, p<0.027\right.$, Fig. 5e). Within the contralateral SNc, significantly fewer THir neurons were observed 5 months following $\alpha$-syn PFF injection $\left(F_{(5,36)}=5.782, p<0.013\right)$ with a non-significant reduction in the contralateral SNc observed at 6 months $(p>0.05)$. A positive correlation existed between the extent of ipsilateral THir SNc neuron loss and the extent of contralateral loss of THir SNc neurons $(r=0.8855$, $p=0.0007, R^{2}=0.7842$, Fig. 3f).

Lastly, to confirm whether reductions in THir neurons induced by PFF injection represented phenotype loss or overt degeneration, unbiased stereology of NeuN-ir neurons in the SNc was conducted in PFF- or PBS-treated groups at 5 and 6 months p.i. No significant differences were observed within the corresponding hemisphere between 5 and 6 months due to either PBS or PFF injection (PBS: $F_{(3,4)}=1.238, p>0.05$; PFF: $F_{(3,8)}=0.3986$, $p>0.05)$. Therefore, the 5- and 6-month time points were combined into one time point. The number of SNc NeuN-ir neurons ipsilateral to PFF injection was significantly reduced compared to either the ipsilateral or contralateral hemisphere of PBS-injected rats $\left(F_{(3,16)}=\right.$ $7.089, p<0.02)$. The number of NeuN-ir neurons in the contralateral SNpc of PFF injected rats was significantly reduced compared to the ipsilateral SNc of PBS injected rats $(p<0.0319)$. When compared to the contralateral $\mathrm{SNc}$ of PBS-injected rats, NeuN-ir neurons were reduced yet did not reach significance $(p=0.0563$, Fig. $3 g-i)$. Overall, our results replicate our previous findings that intrastriatal $\alpha$-syn PFF injection results in significant bilateral reductions in THir and NeuN-ir SNc neurons over the course of 6 months [37].
In a control experiment, we examined whether intrastriatal injection of an exogenous protein taken up by neurons [51, 52], rat serum albumin (RSA), induced an inflammatory response in the absence of intracellular pSyn accumulation. To rule out acute toxicity induced by RSA, THir SNc neurons were quantified at 2 months after injection. No significant differences in THir SNc neurons were observed due to PBS, RSA, or PFF injection in either the ipsilateral or contralateral hemisphere $\left(F_{(5,26)}=0.3731, \quad p>0.05\right.$, Fig. 3j). These results demonstrate that RSA injection, used as an additional control treatment, did not compromise the survival of THir SNc neurons.

\section{Phosphorylated a-syn inclusions peak in the SNc at 2 months and significantly decrease in number during the 5-6-month interval of SNc degeneration}

We determined the time course (1-6 months p.i.) of phosphorylated $\alpha$-syn (pSyn) accumulation in the SNc following intrastriatal $\alpha$-syn PFF injection at monthly intervals. pSyn inclusions were observed in the SNc ipsilateral to injection in all $\alpha$-syn PFF-injected rats, with the number of inclusions varying based on time point after injection (Fig. 4a-d). Inclusions were most abundant at months 1,2, and 3, with all three time points exhibiting significantly higher $\alpha$-syn inclusions compared to the interval of SNc degeneration at months 4,5 , and 6 (Figs. 3e, $g$ and $4 \mathrm{~d} ; F_{(5,18)}=2.251, p \leq 0.001$ ). The number of intraneuronal $\alpha$-syn inclusions in the SNc was significantly greater at 2 months p.i. compared to all other time points except the 1 -month time point (Fig. 4d; $p \leq 0.006$ ). At 2 months, approximately $2220 \pm 148.6 \mathrm{SNc}$ neurons possessed pSyn inclusions. By comparison, a loss of $\approx 3804$ THir SNc neurons ipsilateral to PFF injection was observed at 5-6 months. These results suggest that pSyn inclusion formation in the SNc between 1 and 3 months after PFF injection precedes degeneration of the SNc neurons at 5-6 months p.i.

\section{MHC-II immunoreactive (MHC-Ilir) microglia increase in the SNc in association with accumulation of a-syn inclusion but are decreased during the interval of degeneration}

MHC-II expression on microglia is associated with co-expression of pro-inflammatory genes such as TNF, IL-1 $\beta$, and CD80 as well as proinflammatory cytokine secretion [13-15]. We quantified MHC-II immunoreactive (MHC-IIir) microglia within an adjacent series of SNc tissue sections at months $1,2,3,4,5$, and 6 after unilateral $\alpha$-syn PFF or PBS intrastriatal injection in order to examine neuroinflammation. Double labeling for MHC-II protein and Iba-1 mRNA confirmed the identity of MHC-IIir cells to be microglia (Fig. 4e). 


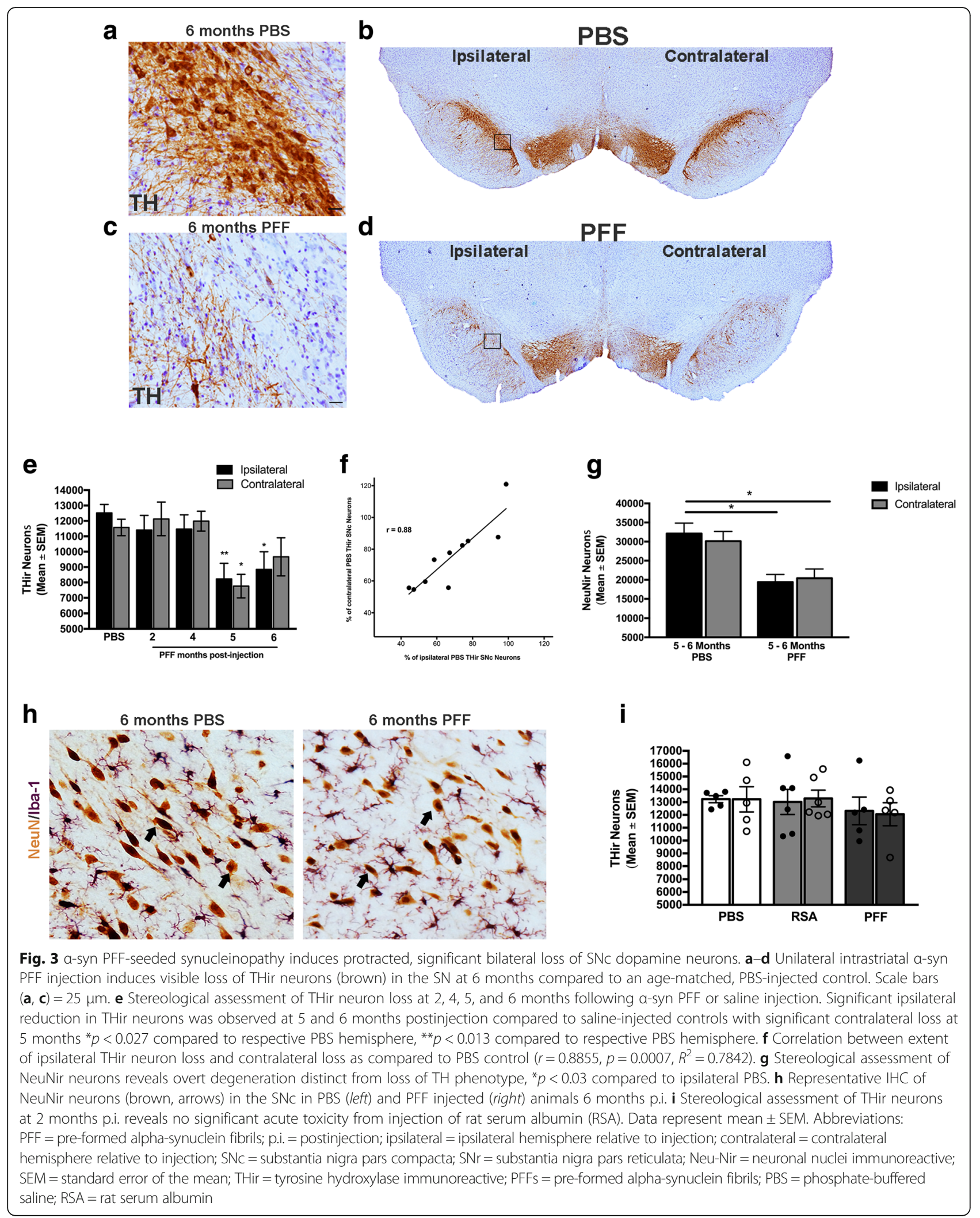



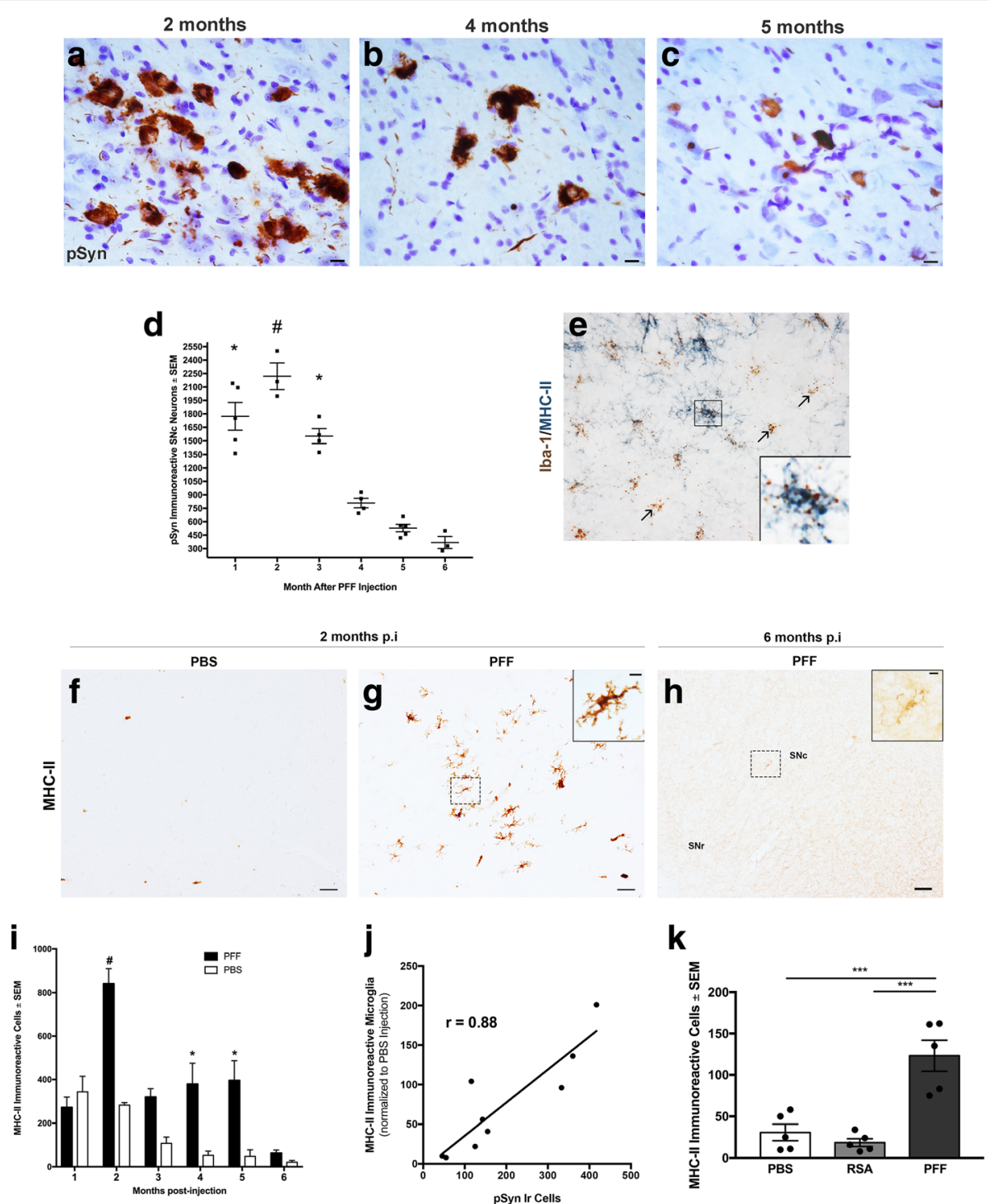

Fig. 4 Antigen-presenting MHC-II immunoreactive (MHC-llir) microglia increase in the SNc in association with peak accumulation of a-syn inclusions but are limited during the interval of degeneration. a-c Representative images of pSyn inclusions in the SNc at 2, 4, and 5 months p.i.; scale bar $(\mathbf{a}-\mathbf{c})=10 \mu \mathrm{m}$. d Stereological assessment of pS129 containing neurons in the substantia nigra in PFF animals at 1, 2, 3, 4, 5, and 6 months p.i.; ${ }^{*} p \leq 0.001$ compared to 4, 5, and 6 months. ${ }^{*} p \leq 0.006$ compared to 3, 4, 5, and 6 months. pSyn inclusions decrease over time in association with neuronal loss. e Major-histocompatibility complex-II (MHC-II; blue) protein colocalizes with ionized calcium-binding adaptor molecule 1 mRNA (brown) within microglia. $\mathbf{f}, \mathbf{g}$ Representative images of MHC-II antigen-presenting microglia in the SN at 2 months in PBS- and PFF-injected rats and 6 months post-PFF injection (h); scale bar $(\mathbf{f}-\mathbf{h})=50 \mu \mathrm{m}$, insets $=10 \mu \mathrm{m}$. i Stereological assessment of MHC-llir microglia in the SN reveals MHC-llir microglia are significantly higher in PFF vs. PBS animals at 2, 4, and 5 months ${ }^{*} p<0.006$. More MHC-llir microglia are evident in 2-month PFF animals vs. all other PFF time points ${ }^{\#} p<0.02$. Notably, MHC-llir microglia peak at the same time pSyn aggregation peaks (d, i). j Number of MHC-II immunoreactive microglia correlated with number of SNc neurons with intraneuronal pSyn inclusions $(r=0.8858$, $p=0.0015, R^{2}=0.7846$ ). $\mathbf{k}$ In a follow-up study, intrastriatal injection of rat serum albumin (RSA) does not impact numbers of MHC-llir microglia compared to PBS $p>0.05$. Injection of PFFs in this second cohort confirmed previous observations of a significant increase in MHC-llir microglia compared to PBS or RSA at 2 months p.i. *** $p \leq 0.0006$. Abbreviations: p.i. = postinjection; PFFs = pre-formed alpha-synuclein fibrils; PBS = phosphate-buffered saline; RSA = rat serum albumin; MHC-II = major-histocompatibility complex-II; Iba-1 = ionized calcium binding adaptor molecule 1; ir = immunoreactive; $\mathrm{SNC}=$ substantia nigra pars compacta; $\mathrm{SEM}=$ standard error of the mean

MHC-IIir microglia were observed in the SNc ipsilateral to injection in both $\alpha$-syn PFF and PBS control rats at all time points. No MHC-IIir microglia were observed in the contralateral SNc. However, the magnitude of
MHC-IIir microglia varied over time and followed a nearly identical pattern to that observed with pSyn inclusion accumulation. At the one-month time point, no significant differences were observed between the 
number of MHC-IIir microglia in the ipsilateral SNc of PBS controls compared to $\alpha$-syn PFF-injected rats $\left(F_{(11,45)}=17.45, p>0.05\right)$, presumably reflecting a nonspecific response to injection (Fig. 4i). However, significantly higher numbers of MHC-IIir microglia were observed in the ipsilateral $\mathrm{SN}$ of $\alpha$-syn PFF-injected rats compared to PBS-injected control rats at months 2, 4, and 5 ( $p<0.006$, Fig. 4f, g, i). The peak of MHC-IIir microglia occurred in the SN 2 months following $\alpha$-syn PFF injection $(p<0.02$ compared to PFF-injected rats all other time points), corresponding to the time point when the greatest number of SNc neurons possesses $\alpha$-syn aggregates (Fig. 4d, g, i). In contrast, significantly fewer MHC-IIir microglia were observed in PFF-injected rats at months 5 and 6, corresponding to the interval of SNc THir neuron loss, although these numbers were still significantly higher than PBS-injected controls $(p<0.006$, Figs. 3e and $4 \mathrm{i})$. There was a positive correlation between the number of MHC-IIir microglia and the number of SNc neurons possessing pS129 $\alpha$-syn inclusions in the $\mathrm{SN}(r=0.8858$, $p=0.0015, R^{2}=0.7846$, Fig. $4 \mathrm{j}$, months 2,4 , and 6).

To confirm these findings, we repeated injections in a separate cohort of animals with rat serum albumin (RSA) as an additional control group for neuronal uptake of exogenous protein in the absence of pSyn accumulation, as PBS injection only controls for needle insertion into the parenchyma. As in previous cohorts, $\alpha$-syn PFF injection resulted in a significant increase in MHC-IIir microglia in the SN at 2 months p.i. (Fig. 4k, $p \leq 0.0006)$. Injection of RSA resulted in similar numbers of MHC-IIir microglia as observed in PBS-injected control rats. No acute neurotoxicity was observed in RSAinjected animals at 2 months p.i. (Fig. 3i). Collectively, these results reveal that the preponderance of MHC-II expression in SN microglia is associated with pSyn $\alpha$-syn inclusions at early time points, however is significantly attenuated during the interval of THir SNc degeneration.

\section{pSyn inclusions in the SNc are associated with a reactive microglial morphology in the adjacent SNr}

The number and distribution of MHC-IIir microglia in the SN suggested that not all microglia were expressing MHC-II. We next used Iba-1 immunoreactivity to examine the entire microglia population within an adjacent series of SN tissue sections at 2 and 6 months after $\alpha$ syn PFF, RSA, or PBS intrastriatal injection (Fig. 5). Quantitation of the number of Iba-1 immunoreactive (Iba-1ir) microglia in the adjacent $\mathrm{SNr}$ revealed no significant differences in microglial number due to $\alpha$-syn PFF, RSA, or PBS injection at either 2 months (Fig. 5a) or 6 months p.i. $\left(2\right.$ months: $F_{(3,16)}=0.2637, p>0.05$; 6 months: $\left.F_{(3,10)}=0.2427, p>0.05\right)$. No significant differences were observed in microglial soma area in the $\mathrm{SNr}$ due to intrastriatal RSA injections at the 2-month time point $\left(F_{(3,16)}=0.256, p=0.855\right.$, Fig. $\left.5 b\right)$. At the 2 -month time point coinciding with the peak of pSyn $\alpha$-syn inclusion accumulation in the SNc, we observed an appreciable increase in the soma size and thickness and number of microglial processes in the SNr of PFF-injected rats compared to a more classically quiescent microglial morphology observed in control injected rats. Specifically, in the ipsilateral $\mathrm{SNr}$ of rats 2 months following $\alpha$-syn PFF injection, the average microglia cell body area was significantly larger compared to PBS-injected rats $\left(F_{(3,16)}=4.016\right.$, $p=0.02$, Fig. $5 \mathrm{c}-\mathrm{e})$. Microglia soma area varied in all conditions between $\approx 10-200 \mu \mathrm{m}^{2}$, with a significantly greater percentage of microglia $>70 \mu \mathrm{m}^{2}$ observed in the $\mathrm{SNr}$ of rats possessing $\mathrm{SNc}$ pSyn $\alpha$-syn inclusions at 2 months compared to rats injected with PBS (Fig. $5 \mathrm{f}-\mathrm{h}$, $\left.F_{(2,12)}=4.613, p=0.03\right)$.

At 6 months p.i., a time point corresponding to the time point of very few pSyn $\alpha$-syn inclusions and immediately following loss of SNc neurons, no significant differences in microglia soma size were observed between $\alpha$-syn PFF- and PBS-injected rats $\left(F_{(3,10)}=2.089\right.$, $p>0.05$, Fig. $5 \mathrm{i}-\mathrm{m}$ ). Of note, the average microglial soma area in PBS-injected rats at 6 months (rats 8 months of age) was significantly larger than PBS-injected rats at 2 months (4 months of age) suggesting an age-related increase $\left(F_{(3,12)}=37.00, p<0.001\right)$. The distribution of microglia soma areas between PFF and PBS rats 6 months following injection also appeared similar (Fig. 5l, m) with an apparent age-related effect [53-55] reflected in a greater percentage of microglia $>70 \mu \mathrm{m}^{2}$ in 8-month-old rats compared to 4-month-old rats.

Overall, our finding that the peak time point of SNc pSyn $\alpha$-syn inclusions is associated with a significant increase in microglia soma size suggests that synucleinopathy in the SNc triggers early disturbances in local microglia. The interval in which we observe this synucleinopathy-induced reactive microglial morphology is 3 months prior to loss of SNc neurons (Fig. 3e, g) suggesting that reactive microglia have the potential to contribute to vulnerability of $\mathrm{SNc}$ neurons to degeneration.

We also examined a series of sections throughout the $\mathrm{SN}$ and striatum at 2, 4, and 6 months p.i. in $\alpha$-syn PFFand PBS-injected rats for the presence of cluster of differentiation 68 (CD68) which labels both phagocytic microglia and infiltrating macrophages. While a few CD68-ir cells were observed in blood vessels, no CD68-ir cells were observed in the parenchyma during any of the time points examined (data not shown). The lack of CD68 immunoreactivity in the parenchyma of the SN or striatum at any time point suggests that the magnitude of synucleinopathy and subsequent degeneration produced in the $\alpha$-syn PFF model does not trigger microglial phagocytic activity. 


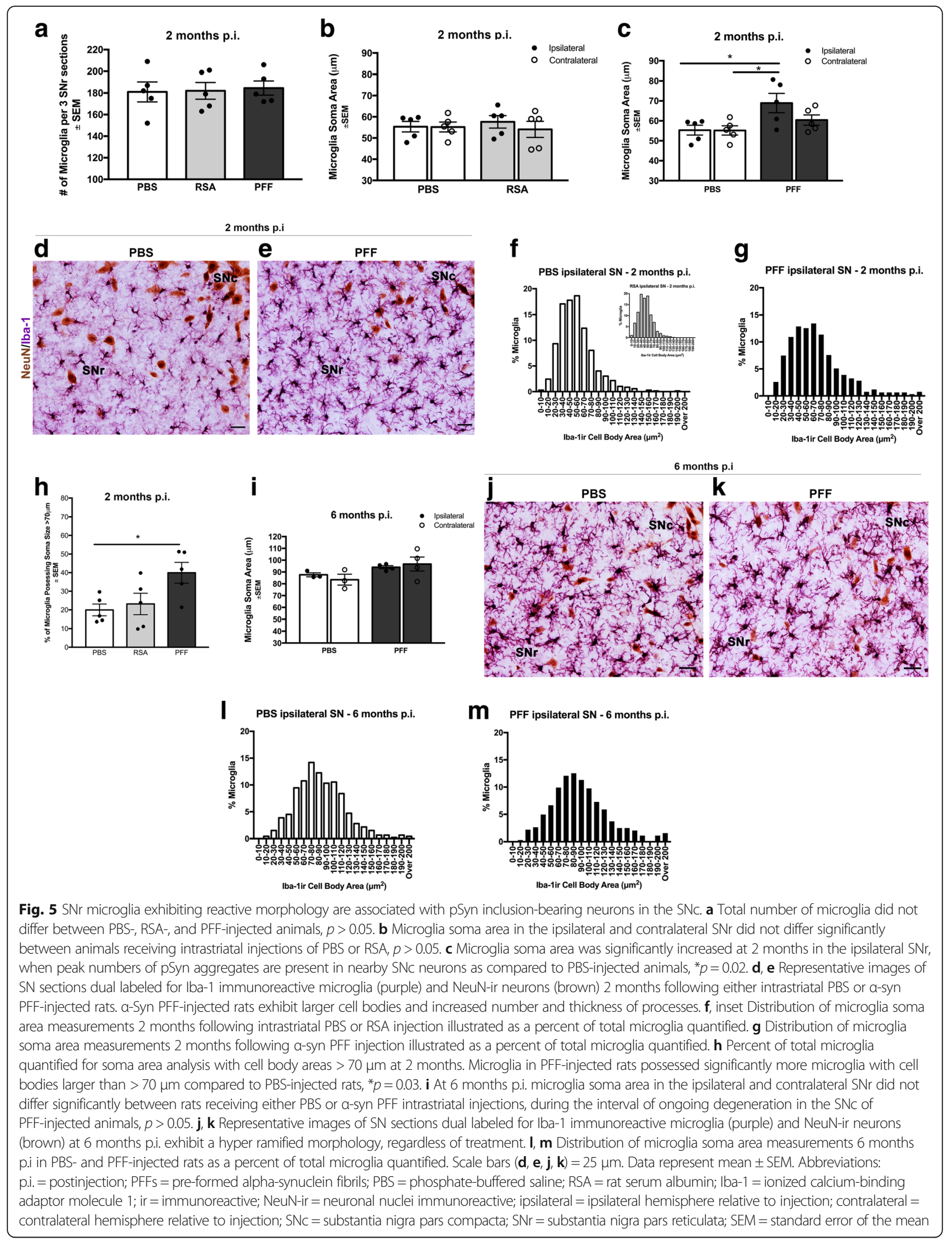


MHC-llir microglia in the agranular insular cortex are associated with the accumulation of a-syn inclusions We also examined the time course of pSyn inclusion accumulation and MHC-II expression on microglia in the agranular insular cortex, as this region possesses abundant Lewy-body like pathology in our model and is implicated in non-motor symptoms in PD [56]. At 2 months p.i., we observe abundant pSyn inclusions primarily localized to the somata (Fig. 6a), with increased neuritic pathology evident by 4 months (Fig. 6b). Interestingly, an observable decrease in both neuritic and somata inclusions is evident by 6 months (Fig. 6c). We observe a similar temporal pattern of MHC-IIir microglia as described for the $\mathrm{SN}$ : the highest number of MHC-IIir microglia is observed at 2 months p.i., when pSyn inclusions first peak, and a decrease in MHC-IIir microglia in association with reductions in the number of pSyn inclusions. Interestingly, a decrease in MHC-IIir microglia is observed between 2 and 4 months p.i. when pSyn pathology becomes more abundant with the appearance of neuritic inclusions. These observations suggest that MHC-II is upregulated as a first response to formation of pSyn inclusions and is not sustained over time, despite a secondary increase in synuclein burden. Few to no MHC-IIir microglia were observed in PBSinjected animals (Fig. $6 \mathrm{~g}-\mathrm{l}$ ), strengthening the concept that MHC-IIir on microglia is induced by initial accumulation of pSyn.

\section{MHC-Ilir microglia in the striatum are not associated with the accumulation of a-syn inclusions}

Lastly, we examined the time course of accumulation of pSyn inclusions and number of MHC-IIir microglia in the striatum in rats that received unilateral $\alpha$-syn PFF or PBS intrastriatal injection. As reported previously [37], the pattern of pSyn $\alpha$-syn inclusion accumulation in the striatum is strikingly different from accumulation in the SNc. At 2 months, $\alpha$-syn inclusions in cell bodies in the ipsilateral striatum are relatively sparse and pSyn $\alpha$-syn immunoreactivity appeared primarily localized to neurites, presumably in terminals from the SNc (Additional file 1: Figure S1A). Over the course of the 4 months, pSyn $\alpha$-syn inclusions in cell bodies in the striatum increase in number, involving the contralateral hemisphere as well, with the greatest number of pSyn $\alpha$-syn inclusions observed in the ipsilateral striatum at the 6-month time point (Additional file 1: Figure S1C). In adjacent striatal tissue sections, we examined the temporal pattern of MHC-IIir microglia following unilateral $\alpha$-syn PFF or PBS intrastriatal injection. Early after injection, at 2 weeks, 1 month, and 2 months, abundant MHC-IIir microglia were observed in the ipsilateral striatum in proximity to the injection sites in both PFF- and PBS-injected rats (Additional file 1: Figure S1D, G). No differences were observed between treatment groups at the 1-month time point, but the magnitude of the MHC-II response appeared slightly larger in PFF-injected rats compared to the PBS-injected rats at the 2-month time point. However, at 4 and 6 months, during the interval of continuing accumulation of pSyn $\alpha$-syn inclusions in the striatum, the number of MHC-IIir microglia decreased dramatically with no differences observed in the small number of MHC-IIir microglia observed in both treatment groups (Additional file 1: Figure S1E, F, H, I). These results suggest that the acute microglial response to the PFF injectate may differ from the acute response to the surgical injection alone but that the subsequent increase $\alpha$-syn inclusion load within neurons in the striatum does not trigger a second wave of microglial MHC-II immunoreactivity.

\section{Discussion}

In the present study, intrastriatal injection of $\alpha$-syn PFFs to rats resulted in widespread accumulation of phosphorylated $\alpha$-syn inclusions in several areas that innervate the striatum, as previously reported in rats and mice $[37,57]$. Further examination of the inclusions formed in the SNc revealed that they share many key features with Lewy bodies and were most abundant between months 1-3 after intrastriatal $\alpha$-syn PFF injection, peaking at 2 months. The magnitude of ipsilateral SNc neurons bearing $\alpha$-syn inclusions $1-3$ months after $\alpha$-syn PFF injection approximated the magnitude of loss of ipsilateral SNc neurons observed at 5-6 months, suggesting a direct relationship between $\alpha$-syn inclusion accumulation and degeneration of SNc neurons. Synucleinopathy-specific MHC-II expression in the ipsilateral SNc similarly peaked in the $\mathrm{SN}$ at 2 months and was associated with a reactive microglial morphology, characterized by significantly larger soma size, 3 months prior to degeneration. Surprisingly, although the period of nigral degeneration was associated with an increased MHC-II signal relative to controls, MHC-II immunoreactivity during the period of degeneration was significantly decreased relative to MHC-II immunoreactivity during the earlier peak of synucleinopathy. Overall, the temporal pattern of peak Lewy body-like inclusion formation was associated with peak neuroinflammation in the $\mathrm{SN}$, both of which appear months prior to loss of SNc neurons. These results suggest that an increase in MHC-II may be a first-response mechanism to initial accumulation of intracellular $\alpha$-syn and that reactive microglia have the potential to contribute to vulnerability of SNc neurons to degeneration (Fig. 7, left).

Within the agranular insular cortex, we observe pSyn primarily localized to the somata at 2 months, followed by an increase in neuritic pathology by 4 months, and a significant decrease overall in pSyn immunoreactivity at 6 months (Fig. 7, right). This suggests that similar to the $\mathrm{SN}$, neurons in the agranular insular cortex harboring 


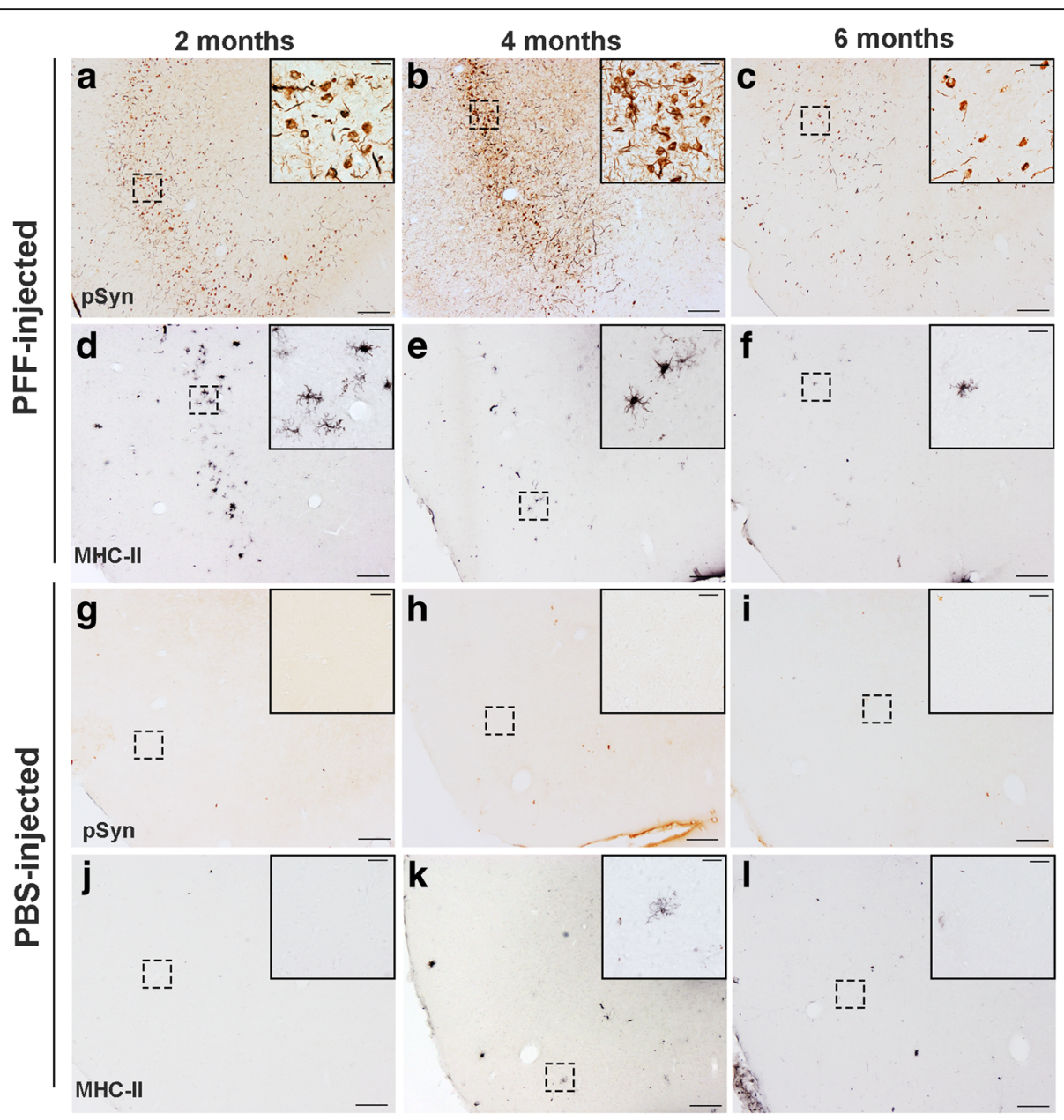

Fig. 6 Microglia expressing MHC-II are associated with pSyn inclusions in the agranular insular cortex. a-c Representative images of pSyn accumulation in the agranular insular cortex at 2, 4, and 6 months p.i. Pathology is primarily localized to the soma at 2 months (a), with increased neuritic pathology by 4 months (b) and an observable decrease overall by 6 months, possibly due to death of neurons. c No pSyn inclusions were observed in PBS-injected animals ( $\mathbf{g}-\mathbf{i})$. $\mathbf{d}-\mathbf{f}$ A similar pattern was observed in MHC-Il expression on microglia. Peak numbers of MHC-llir microglia were observed at 2 months p.i. (d), decreased at 4 months (e), and virtually absent by 6 months (f). j-I Few to no MHC-llir microglia were observed in PBS injected animals at any time point, suggesting that MHC-II expression occurs in response to accumulation of pSyn inside neurons. Abbreviations: p.i. = postinjection; pSyn = $a-s y n$ phosphorylated at serine 129; PFFs = pre-formed alpha-synuclein fibrils; PBS = phosphate-buffered saline; MHC-II= major-histocompatibility complex-II; ir = immunoreactive

inclusions die over the course of 6 months. Moreover, a similar pattern of MHC-II expression on microglia is observed: numbers of MHC-IIir microglia peak when pSyn pathology is most abundant and decrease over time. Although this has not been systematically quantified in the present study, future studies investigating inflammation and degeneration in this area following intrastriatal $\alpha$ syn PFF injection are warranted, as the insula has been implicated in the manifestations of non-motor symptoms in human PD $[56,58,59]$.

Within the striatum, the site of $\alpha$-syn PFF injection, we observed a distinctly different pattern of accumulation of $\alpha$-syn inclusions compared to the SNc (Additional file 3: Figure S3J). We observed a dissociation between $\alpha$-syn inclusion load and MHC-II immunoreactivity in the striatum (Additional file 3: Figure S3). The procedure of intrastriatal injection itself triggered an acute increase in MHC-II immunoreactive microglia that appeared to be slightly enhanced by the presence of the PFF injectate; however, the presence of MHC-II decreased dramatically over time in all conditions despite an ever increasing $\alpha$ syn inclusion load. Compared to the SNc, the accumulation of $\alpha$-syn inclusions in the striatum is delayed with no loss of striatal neurons observed at 6 months [37] and it is unknown whether degeneration of striatal neurons ultimately occurs at later time points. It is unclear whether $\alpha$ syn inclusion load increases and peaks past the 6-month time point and whether the presence of MHC-II immunoreactive microglia may have similarly tracked with a future peak. The results in the striatum illustrate the necessity of 


\section{Time Course of a-syn Inclusions and Neuroinflammation}
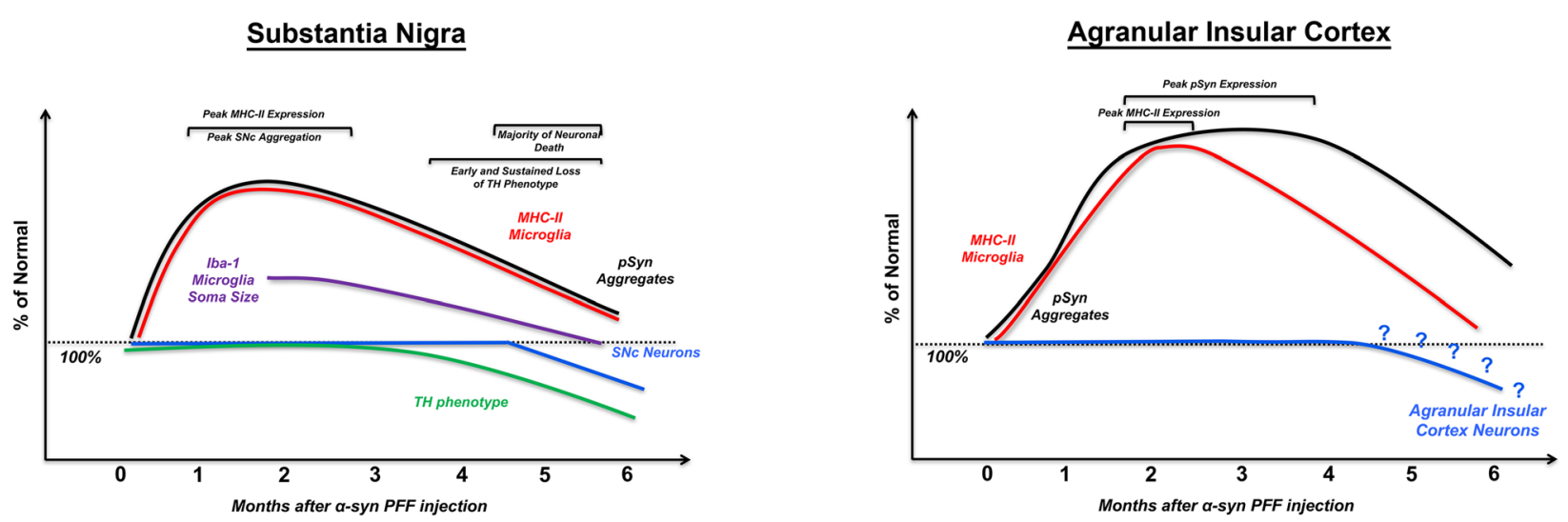

Fig. 7 Regional timelines of synucleinopathy, neuroinflammation, and degeneration in the substantia nigra and agranular insular cortex following intrastriatal a-syn PFF injection. (Left): Early accumulation of phosphorylated inclusions of a-syn (peak at 2 months) in the substantia nigra leads to loss of TH phenotype and eventual loss of nigrostriatal dopamine neurons by 5-6 months p.i. In the SN, the pattern of microgliosis similarly follows that of pSyn: microglia in the adjacent SNr exhibit a reactive morphology at 2 months p.i. when nearby SNc neurons possess the greatest number of SNc pSyn inclusions. Interestingly, MHC-llir antigen-presenting microglia in the SNc also peak at 2 months p.i., again coinciding with the greatest number of pSyn intraneuronal inclusions and decrease over time to near non-detectable levels during the interval of degeneration, suggesting a relationship between pathological a-syn and inflammation. (Right): Early accumulation of pSyn inclusions occurs between 2 and 4 months, with inclusions primarily localized to the somata at 2 months, an increase in neuritic inclusions at 4 months, and an observable decrease of overall pSyn pathology at 6 months, suggesting that similar to the SN, neurons in the agranular insular cortex harboring inclusions eventually die off. MHC-II immunoreactivity follows a similar pattern to that observed in the SN, with peak expression observed at 2 months p.i., and decreased over the course of 6 months

determining the role of an acute inflammatory response to local injection and that the magnitude of this response may prevent the reactive microgliosis associated with pathological $\alpha$-syn inclusions. Further, these striatal results suggest that $\alpha$-syn inclusions do not automatically trigger reactive microgliosis and that other factors including rate of inclusion formation, impending cytotoxicity, local environment, or microglia and astrocyte density [42] may be involved in determining the neuroinflammatory cascade of events.

In response to persistent neuronal stress and protein accumulation such as $\alpha$-syn aggregation, microglia can become chronically activated, proliferate, migrate, secrete pro-inflammatory cytokines and reactive oxygen species, and ultimately contribute to neuronal injury in an uncontrolled, feed-forward manner [20, 60-63]. Microglia can also phagocytose both living and dead neurons [64-66]. The observation that $\alpha$-syn inclusion triggered MHC-II expression on microglia in the SN prior to degeneration indicates that neuroinflammation may contribute to the mechanism of pathophysiology. However, it is unlikely that microglial activation is the sole arbiter of degeneration given that neuronal death can result from $\alpha$-syn PFF-induced intraneuronal inclusions in cultures in the absence of microglia [40]. A more likely scenario involves neuroinflammation contributing to or accelerating nigrostriatal degeneration with properties unique to the nigral environment [67-72] adding to the cascade of events. Future studies investigating the secretion of proinflammatory and anti-inflammatory cytokines in the $\mathrm{SN}$ at time points prior to and following nigral degeneration is warranted.

Human PD studies examining neuroinflammation suggest involvement of both the local brain immune response and the adaptive immune system in PD [73-75]. In the present study, we did not examine the possibility of peripheral immune cell infiltration beyond phagocytic CD68+ macrophages, which were not detected in the parenchyma in PFF-injected animals at any stage, and data from human tissue regarding the presence of $\mathrm{CD} 68+$ macrophages in human PD is limited [9]. However, microglia are considered to be the principle antigen-presenting cell within the brain and MHC-II expression is associated with the recognition of $\mathrm{CD} 4+\mathrm{T}$-helper cells. It is possible that $\mathrm{CD} 4+\mathrm{T}$ cells participate in the response to $\alpha$-syn inclusions in the $\mathrm{SN}$; however, whether the net effect of CD4+ $\mathrm{T}$ cells is neurodegenerative or neuroprotective requires further systematic evaluation [76]. In addition, MHC-I expression by SNc neurons was observed in association with $\alpha$-syn overexpression [77] and may similarly be expressed by $\alpha$-syn inclusion-bearing SNc neurons. In addition, it was recently shown that $\mathrm{T}$ lymphocytes isolated from $\mathrm{PD}$ patients recognize specific $\alpha$-syn peptides [78], strengthening the concept that neuroinflammation can be induced by $\alpha$-syn and potentially involved early in PD progression.

While the concept that MHC-II is involved in PD and correlates with $\alpha$-syn burden [9] has existed for several 
decades, our results are the first to systematically evaluate the time course of endogenous pSyn accumulation and microglial MHC-II expression prior to and after nigrostriatal degeneration has occurred. Our results indicate that MHC-II expression in the SNc is increased at early time points in which SNc neurons possess pSyn inclusions, and is relatively sparse during the interval of nigral degeneration, suggesting that MHC-II is a response to initial inclusion formation. Future studies investigating the direct impact of increased or decreased MHC-II expression on the magnitude of degeneration and what role, if any, peripheral $\mathrm{T}$ cells play in disease progression are warranted.

Our results and the well-characterized rat $\alpha$-syn PFF model will facilitate future studies to provide key mechanistic insights into the specific relationship between pathological $\alpha$-syn inclusions, neuroinflammation, and degeneration in sporadic PD.

\section{Conclusions}

Accumulation of intraneuronal inclusions of phosphorylated $\alpha$-syn induces increased MHC-II expression and reactive microgliosis in the substantia nigra months prior to dopaminergic cell death, suggesting that microglia may be a contributor to rather than only a consequence of nigral degeneration. These results will facilitate future studies to provide key mechanistic insights into the specific relationship between pathological $\alpha$-syn inclusions, neuroinflammation, and degeneration in sporadic PD.

\section{Additional files}

Additional file 1: Figure S1. Unilateral intrastriatal injection of a-syn PFFs, but not RSA or PBS, induces bilateral cortical and unilateral SNC Lewy-body like inclusions of phosphorylated a-syn (pSyn). (a) pSyn pathology is observed bilaterally in cortical areas after unilateral injection of a-syn PFFs, namely in layers 2/3 and orbital and agranular insular cortices. (b) Injection of PBS or (c) RSA did not induce pSyn accumulation. (d) pSyn accumulation in the ipsilateral substantia nigra pars compacta (SNc) at 2 months postinjection, with no evidence of pSyn inclusions in the contralateral SNc. Scale bars $(A-D)=50 \mu \mathrm{m}$. Abbreviations: $a-$ syn = alphasynuclein; PFFs = pre-formed alpha-synuclein fibrils; PBS = phosphatebuffered saline; RSA = rat serum albumin; $\mathrm{pSyn}=\mathrm{a}$-syn phosphorylated at serine 129. (TIF $117729 \mathrm{~kb})$

Additional file 2: Figure S2. Unilateral intrastriatal injection of a-syn PFFs induces widespread accumulation of Lewy-body like inclusions of phosphorylated a-syn (pSyn). Representative images illustrating the time course of pSyn pathology in regions innervating the striatum. $(a-c)$ pSyn pathology in the ipsilateral agranular insular cortex localized to both the soma and neurites at 2 months p.i. (postinjection) that over time becomes primarily localized to the soma; scale bar $=50 \mu \mathrm{m}$, inset $=10 \mu \mathrm{m}$. $(d-f)$ Ipsilateral accumulation of pSyn in the substantia nigra peaks at 2 months and becomes less abundant over time as neurons degenerate; scale bar $=200 \mu \mathrm{m}$, inset $=25 \mu \mathrm{m}$. (g-i) In contrast to other areas, pSyn in the striatum is primarily localized to neurites at 2 months and becomes more abundant and localized to the soma over time, scale bar $=50 \mu \mathrm{m}$, inset $=10 \mu \mathrm{m}$. Abbreviations: $\mathbf{a}$-syn = alpha-synuclein; PFFs = pre-formed alpha-synuclein fibrils; pSyn =a-syn phosphorylated at serine 129; p.i. = postinjection. (TIF $33472 \mathrm{~kb}$ )
Additional file 3: Figure S3. Antigen-presenting MHC-llir microglia are not associated with peak of intraneuronal inclusions of pSyn in the striatum. Progression of pSyn pathology and MHC-llir microglia in the striatum. (a) At 2 months p.i., pSyn inclusions are localized to neurites, presumably representing terminals from the SNc. (b-c) Over time pSyn inclusions become primarily localized to the soma of striatal neurons. (d) Abundant MHC-llir microglia in the striatum primarily localized around the a-syn PFF injection site at 2 months. (e-f) MHC-llir microglia in the striatum are largely absent during continuing accumulation of intraneuronal pSyn inclusions at 4 months (e) and 6 months ( $f$ ) p.i. (g) Intrastriatal injection of PBS results abundant MHC-llir microglia in the striatum localized near the site of injection at 2 months p.i., although appearing less abundant than MHC-llir microglia in the striatum of a-syn PFF rats at the same time point (d). (h) MHC-llir microglia are similarly absent from the parenchyma by 4 months (h) and 6 months p.i (i). Scale bars $A-I=50 \mu \mathrm{m}$. Abbreviations: p.i. = postinjection; PFFs = pre-formed alpha-synuclein fibrils;

PBS = phosphate-buffered saline; pSyn = a-syn phosphorylated at serine 129, MHC-llir = major-histocompatibility complex-II immunoreactive. (TIF 112368 kb)

\section{Abbreviations}

6-OHDA: 6-Hydroydopamine; AAV: Adenoassociated virus; CD68: Cluster of differentiation molecule 68; DMS: Dorsomedial striatum; Iba-1: Ionized calcium-binding adaptor molecule-1; IHC: Immunohistochemistry; MHC-II: Major histocompatibility complex II; MPTP: 1-Methyl-4-phenyl-1,2,3, 6-tetrahydropyridine; NeuN: Neuronal nuclei; PBS: Phosphate-buffered saline; PD: Parkinson's disease; PFFs: Alpha-synuclein pre-formed fibrils; pSyn: Alpha-synuclein phosphorylated at serine 129; RSA: Rat serum albumin; SNc: Substantia nigra pars compacta; SNr: Substantia nigra pars reticulata; TH: Tyrosine hydroxylase; VLS: Ventrolateral striatum; a-syn: Alpha-synuclein

\section{Funding}

A support was provided by the Department of Translational Science and Molecular Medicine, the Neuroscience Graduate Program, National Institute of Neurological Disorders and Stroke (NS099416), and the Edwin A. Brophy Endowment at Michigan State University.

\section{Authors' contributions}

This research project was conceived and organized by MFD, TJC, MGT, KCL, NMK, and CES. It was executed by MFD, CES, TJC, JRP, CJK, KCL, KLP, NMK, DLF, NKP, OLB, JWH, NNV, NKM, and OMAE. The data were analyzed by MFD, CES, and TJC. The manuscript was first written and revised by MFD and CES, and it was reviewed and critiqued by all authors. All authors read and approved the final manuscript.

\section{Ethics approval}

All procedures performed in studies involving animals were in accordance with the ethical standards of the Institute for Animal Use and Care Committee (IACUC) at Michigan State University.

\section{Competing interests}

The authors declare that they have no competing interests.

\section{Publisher's Note}

Springer Nature remains neutral with regard to jurisdictional claims in published maps and institutional affiliations.

\section{Author details}

${ }^{1}$ Department of Translational Science and Molecular Medicine, Michigan State University, 400 Monroe Avenue NW, Grand Rapids, MI 49503-2532, USA. ${ }^{2}$ Neuroscience Graduate Training Program, Michigan State University, Grand Rapids, MI, USA. ${ }^{3}$ MD/PhD Program, Michigan State University, Grand Rapids, MI, USA. ${ }^{4}$ Mercy Health Hauenstein Neuroscience Medical Center, Grand Rapids, MI, USA. ${ }^{5}$ Center for Neurodegenerative Disease Research, Department of Pathology and Laboratory Medicine, University of Pennsylvania Perelman School of Medicine, Philadelphia, PA, USA. ${ }^{6}$ Department of Physiology, Emory University School of Medicine, Atlanta, GA, USA. ${ }^{7}$ Neurological Disorders Research Center, Qatar Biomedical Research Institute (QBRI), Hamad Bin Khalifa University (HBKU), Education City, Qatar. ${ }^{8}$ Life Sciences Division, College of Science and Engineering, Hamad Bin Khalifa University (HBKU), Education City, Qatar. 
Received: 15 March 2018 Accepted: 20 April 2018 Published online: 01 May 2018

\section{References}

1. Collier TJ, Kanaan NM, Kordower JH. Aging and Parkinson's disease: different sides of the same coin? Mov Disord. 2017;32(7):983-90

2. Collier TJ, Kanaan NM, Kordower JH. Ageing as a primary risk factor for Parkinson's disease: evidence from studies of non-human primates. Nat Rev Neurosci. 2011;12(6):359-366

3. Mogi $M$, et al. Interleukin (IL)-1 beta, IL-2, IL-4, IL-6 and transforming growth factor-alpha levels are elevated in ventricular cerebrospinal fluid in juvenile parkinsonism and Parkinson's disease. Neurosci Lett. 1996;211(1):13-6.

4. Lindqvist $D$, et al. Cerebrospinal fluid inflammatory markers in Parkinson's disease-associations with depression, fatigue, and cognitive impairment. Brain Behav Immun. 2013;33:183-9.

5. Gerhard A, et al. In vivo imaging of microglial activation with [11C](R)-PK11195 PET in idiopathic Parkinson's disease. Neurobiol Dis. 2006;21(2):404-12.

6. McGeer PL, et al. Reactive microglia are positive for HLA-DR in the substantia nigra of Parkinson's and Alzheimer's disease brains. Neurology. 1988:38(8):1285-91.

7. Imamura K, et al. Distribution of major histocompatibility complex class II-positive microglia and cytokine profile of Parkinson's disease brains. Acta Neuropathol. 2003;106(6):518-26.

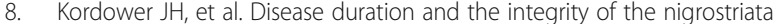
system in Parkinson's disease. (1460-2156 (Electronic)).

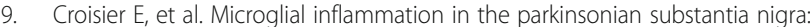
relationship to alpha-synuclein deposition. J Neuroinflammation. 2005;2:14.

10. Dijkstra AA, et al. Evidence for immune response, axonal dysfunction and reduced endocytosis in the substantia nigra in early stage Parkinson's disease. PLoS One. 2015;10(6):e0128651.

11. Braak $H$, et al. Staging of brain pathology related to sporadic Parkinson's disease. Neurobiol Aging. 2003;24(2):197-211.

12. Kannarkat GT, et al. Common genetic variant association with altered HLA expression, synergy with Pyrethroid exposure, and risk for Parkinson's disease: an observational and case-control study. NPJ Parkinsons Dis. 2015;1: 359-66

13. Spiller $\mathrm{KL}$, et al., The role of macrophage phenotype in vascularization of tissue engineering scaffolds. Biomaterials. 2014;35(15): 4477-88.

14. Harms AS, et al. MHCll is required for alpha-synuclein-induced activation of microglia, CD4 T cell proliferation, and dopaminergic neurodegeneration. J Neurosci. 2013:33(23):9592-600.

15. Liu X, et al. Intracellular MHC class II molecules promote TLR-triggered innate immune responses by maintaining activation of the kinase Btk. Nat Immunol. 2011;12:416

16. Cicchetti F, et al. Neuroinflammation of the nigrostriatal pathway during progressive 6-OHDA dopamine degeneration in rats monitored by immunohistochemistry and PET imaging. Eur J Neurosci. 2002;15(6):991-8.

17. Koprich JB, et al. Neuroinflammation mediated by IL-1 beta increases susceptibility of dopamine neurons to degeneration in an animal model of Parkinson's disease. J Neuroinflammation. 2008:5:8.

18. Forno LS, et al. Similarities and differences between MPTP-induced parkinsonism and Parkinson's disease. Neuropathologic considerations. Adv Neurol. 1993;60:600-8

19. Kurkowska-Jastrzebska I, et al. The inflammatory reaction following 1-methyl-4-phenyl-1,2,3, 6-tetrahydropyridine intoxication in mouse. Exp Neurol. 1999;156(1):50-61.

20. Gao HM, et al. Neuroinflammation and alpha-synuclein dysfunction potentiate each other, driving chronic progression of neurodegeneration in a mouse model of Parkinson's disease. Environ Health Perspect. 2011;119(6):807-14.

21. Gomez-Isla T, et al. Motor dysfunction and gliosis with preserved dopaminergic markers in human alpha-synuclein A30P transgenic mice. Neurobiol Aging. 2003;24(2):245-58.

22. Koprich JB, et al. Expression of human A53T alpha-synuclein in the rat substantia nigra using a novel AAV1/2 vector produces a rapidly evolving pathology with protein aggregation, dystrophic neurite architecture and nigrostriatal degeneration with potential to model the pathology of Parkinson's disease. Mol Neurodegener. 2010;5:43.

23. Yamada M, Iwatsubo $T$, Mizuno $Y$, Mochizuki $H$. Overexpression of asynuclein in rat substantia nigra results in loss of dopaminergic neurons, phosphorylation of a-synuclein and activation of caspase-9: resemblance to pathogenetic changes in Parkinson's disease. J Neurosci. 2004; 91:451-61.

24. Ulusoy A, et al., Chapter 5 - Viral vector-mediated overexpression of asynuclein as a progressive model of Parkinson's disease. In: Björklund A and Cenci MA, editors. Progress in Brain Research. Elsevier; 2010. p. 89-111.

25. Theodore $\mathrm{S}$, et al., Targeted Overexpression of Human a-Synuclein Triggers Microglial Activation and an Adaptive Immune Response in a Mouse Model of Parkinson Disease. J Neuropathol Exp Neurol. 2008; 67(12): p. 1149-58.

26. Oliveras-Salvá $M$, et al. rAAV2/7 vector-mediated overexpression of alpha-synuclein in mouse substantia nigra induces protein aggregation and progressive dose-dependent neurodegeneration. Mol Neurodegener. 2013;8:44.

27. Gombash SE, et al. Morphological and behavioral impact of AAV2/5-mediated overexpression of human wildtype alpha-synuclein in the rat nigrostriatal system. PLoS One. 2013:8(11):e81426.

28. Fischer DL, et al., Viral Vector-Based Modeling of Neurodegenerative Disorders: Parkinson's Disease. In: Manfredsson FP, Editor. Gene Therapy for Neurological Disorders: Methods and Protocols. New York: Springer New York; 2016. p. 367-382

29. Simola N, Morelli M, Carta A. The 6-hydroxydopamine model of Parkinson's Disease. 2007:11:151-67.

30. Matsuoka Y, et al. Lack of nigral pathology in transgenic mice expressing human alpha-synuclein driven by the tyrosine hydroxylase promoter. Neurobiol Dis. 2001;8(3):535-9.

31. Ip CW, et al. AAV1/2-induced overexpression of A53T-a-synuclein in the substantia nigra results in degeneration of the nigrostriatal system with Lewy-like pathology and motor impairment: a new mouse model for Parkinson's disease. Acta Neuropathol Commun. 2017:5:11.

32. Sanchez-Guajardo $V$, et al. Microglia acquire distinct activation profiles depending on the degree of alpha-synuclein neuropathology in a rAAV based model of Parkinson's disease. PLoS One. 2010;5(1):e8784.

33. Thakur $P$, Breger LS, Lundblad M, et al. Modeling Parkinson's disease pathology by combination of fibril seeds and a-synuclein overexpression in the rat brain. Proc Natl Acad Sci USA. 2017:114(39):E8284-93

34. Farrer $M$, et al. Comparison of kindreds with parkinsonism and alpha-synuclein genomic multiplications. Ann Neurol. 2004;55(2):174-9.

35. Su X, et al. Alpha-Synuclein mRNA Is Not Increased in Sporadic PD and Alpha-Synuclein Accumulation Does Not Block GDNF Signaling in Parkinson's Disease and Disease. Models Mol Ther.2017; 25(10): p. $2231-$ 2235.

36. Zhou J, et al., Changes in the solubility and phosphorylation of a-synuclein over the course of Parkinson's disease. Acta Neurochir. 2011;121(6): p. 695704.

37. Paumier $\mathrm{KL}$, et al. Intrastriatal injection of pre-formed mouse alpha-synuclein fibrils into rats triggers alpha-synuclein pathology and bilateral nigrostriatal degeneration. Neurobiol Dis. 2015;82:185-99.

38. Luk KC, et al. Intracerebral inoculation of pathological a-synuclein initiates a rapidly progressive neurodegenerative a-synucleinopathy in mice. Jxp Med. 2012;209(5):975-86

39. Polinski NK, et al., Best Practices for Generating and Using Alpha-Synuclein Pre-Formed Fibrils to Model Parkinson's Disease in Rodents. J Parkinsons Dis. 2018

40. Volpicelli-Daley LA, Luk KC, Lee VM. Addition of exogenous alpha-synuclein preformed fibrils to primary neuronal cultures to seed recruitment of endogenous alpha-synuclein to Lewy body and Lewy neurite-like aggregates. Nat Protoc. 2014;9(9):2135-46.

41. Volpicelli-Daley LA, et al. Exogenous alpha-synuclein fibrils induce Lewy body pathology leading to synaptic dysfunction and neuron death. Neuron. 2011;72(1):57-71.

42. Tarutani A, Suzuki G, Shimozawa A, et al. The Effect of Fragmented Pathogenic a-Synuclein Seeds onPrion-like Propagation. The Journal of Biological Chemistry. 2016;291(36):18675-18688

43. Vaikath NN, et al., Generation and characterization of novel conformationspecific monoclonal antibodies for a-synuclein pathology. Neurobiol Dis. 2015; 79: p. 81-99.

44. Leys $C$, et al. Detecting outliers: do not use standard deviation around the mean, use absolute deviation around the median. J Exp Soc Psychol. 2013:49(4):764-6.

45. Leys $C$, et al., Detecting outliers: Do not use standard deviation around the mean, use absolute deviation around the median. J Exp Soc Psychol. 2013; 49(4): p. 764-766. 
46. Kim C, et al. Neuron-released oligomeric alpha-synuclein is an endogenous agonist of TLR2 for paracrine activation of microglia. Nat Commun. 2013:4:1562

47. Wan OW, Chung KK. The role of alpha-synuclein oligomerization and aggregation in cellular and animal models of Parkinson's disease. PLoS One, 2012; 7(6): p. e38545.

48. Winner $B$, et al., In vivo demonstration that alpha-synuclein oligomers are toxic. Proc Natl Acad Sci USA, 2011. 108(10): p. 4194-9.

49. Li JY, et al., Characterization of Lewy body pathology in 12- and 16-year-old intrastriatal mesencephalic grafts surviving in a patient with Parkinson's disease. Mov Disord. 2010. 25(8): p. 1091-6.

50. Tanji K, et al., Proteinase K-resistant alpha-synuclein is deposited in presynapses in human Lewy body disease and A53T alpha-synuclein transgenic mice. Acta Neuropathol. 2010; 120(2): p. 145-54.

51. Liu Z, et al., Neuronal uptake of serum albumin is associated with neuron damage during the development of epilepsy. Exp Ther Med. 2016; 12(2): p. 695-701.

52. Rey NL., et al., Widespread transneuronal propagation of alphasynucleinopathy triggered in olfactory bulb mimics prodromal Parkinson's disease. J Exp Med. 2016; 213(9): p. 1759-78.

53. Conde JR, Streit WJ. Effect of aging on the microglial response to peripheral nerve injury. Neurobiol Aging. 2006; 27(10): p. 1451-61

54. Streit WJ, Microglia and neuroprotection: implications for Alzheimer's disease. Brain Res Brain Res Rev, 2005; 48(2): p. 234-9.

55. Streit WJ, et al., Dystrophic microglia in the aging human brain. Glia. 2004; 45(2): p. 208-12.

56. Christopher $L$, et al., Uncovering the role of the insula in non-motor symptoms of Parkinson's disease. Brain. 2014; 137(Pt 8): p. 2143-54.

57. Luk KC, et al. Pathological alpha-synuclein transmission initiates Parkinson-like neurodegeneration in nontransgenic mice. Science. 2012;338(6109):949-53.

58. Maillet $A$, et al., The prominent role of serotonergic degeneration in apathy, anxiety and depression in de novo Parkinson's disease. Brain 2016; 139(Pt 9): p. 2486-502.

59. Cerasa A., et al., Cortical volume and folding abnormalities in Parkinson's disease patients with pathological gambling. Parkinsonism Relat Disord. 2014. 20(11): p. 1209-14

60. Block ML, Hong JS. Microglia and inflammation-mediated neurodegeneration: multiple triggers with a common mechanism. Prog Neurobiol. 2005;76(2):77-98.

61. Gao HM, et al. Neuroinflammation and oxidation/nitration of alpha-synuclein linked to dopaminergic neurodegeneration. J Neurosci. 2008;28(30):7687-98.

62. Hwang O. Role of oxidative stress in Parkinson's disease. Exp Neurobiol. 2013;22(1):11-7.

63. Wilshusen RA, Mosley RL. Innate and adaptive immune-mediated neuroinflammation and neurodegeneration in Parkinson's disease. In: Peterson PK, Toborek M, editors. Neuroinflammation and neurodegeneration. New York: Springer New York; 2014. p. 119-42.

64. Hong S, Beja-Glasser VF, Nfonoyim BM, et al. Complement and Microglia Mediate Early Synapse Loss in Alzheimer Mouse Models. Science (New York, NY). 2016;352(6286):712-716

65. Solga AC, et al., RNA-sequencing reveals oligodendrocyte and neuronal transcripts in microglia relevant to central nervous system disease. Glia. 2015; 63(4): p. 531-548.

66. Park JY, et al., Microglial phagocytosis is enhanced by monomeric alphasynuclein, not aggregated alpha-synuclein: implications for Parkinson's disease. Glia. 2008; 56(11): p. 1215-23.

67. Farooqui T, Farooqui AA. Lipid-mediated oxidative stress and inflammation in the pathogenesis of Parkinson's disease. Parkinsons Dis. 2011;2011:247467.

68. Kim WG, et al. Regional difference in susceptibility to lipopolysaccharideinduced neurotoxicity in the rat brain: role of microglia. J Neurosci. 2000;20(16):6309-16.

69. Zhang K, Kaufman RJ. From endoplasmic-reticulum stress to the inflammatory response. Nature. 2008;454(7203):455-62.

70. Zhang W, et al. Human neuromelanin: an endogenous microglial activator for dopaminergic neuron death. Front Biosci (Elite Ed). 2013;5:1-11.

71. Andringa $\mathrm{G}$, et al. Mapping of rat brain using the synuclein-1 monoclonal antibody reveals somatodendritic expression of alpha-synuclein in populations of neurons homologous to those vulnerable to Lewy body formation in human synucleopathies. J Neuropathol Exp Neurol. 2003;62(10):1060-75.

72. Urrutia PJ, Mena NP, Núñez MT. The interplay between iron accumulation, mitochondrial dysfunction, and inflammation during the execution step of neurodegenerative disorders. Front Pharmacol. 2014;5:38.

73. Brochard V, et al., Infiltration of CD4+ lymphocytes into the brain contributes to neurodegeneration in a mouse model of Parkinson disease. J Clin Invest. 2009. 119(1): p. 182-92.

74. Jiang S, et al., The correlation of lymphocyte subsets, natural killer cell, and Parkinson's disease: a meta-analysis. Neurol Sci. 2017; 38(8): p. 1373-1380.

75. Kustrimovic N, et al., Dopaminergic Receptors on CD4+ T Naive and Memory Lymphocytes Correlate with Motor Impairment in Patients with Parkinson's Disease. Sci Rep. 2016; 6: p. 33738.

76. Carson MJ, Thrash JC, Walter B. The cellular response in neuroinflammation: The role of leukocytes, microglia and astrocytes in neuronal death and survival. Clin Neurosci Res. 2006; 6(5): p. 237-45

77. Cebrian $C_{\text {, }}$ et al. MHC-I expression renders catecholaminergic neurons susceptible to T-cell-mediated degeneration. Nat Commun. 2014;5:3633.

78. Sulzer D., et al., T cells from patients with Parkinson's disease recognize alpha-synuclein peptides. Nature. 2017; 546(7660): p. 656-61.

\section{Ready to submit your research? Choose BMC and benefit from:}

- fast, convenient online submission

- thorough peer review by experienced researchers in your field

- rapid publication on acceptance

- support for research data, including large and complex data types

- gold Open Access which fosters wider collaboration and increased citations

- maximum visibility for your research: over $100 \mathrm{M}$ website views per year

At BMC, research is always in progress.

Learn more biomedcentral.com/submissions 